Carolina Barbosa de Souza

\title{
Predição de risco para dificuldades motoras em prematuros com idade pré-escolar pelo General Movement Assessment
}

\author{
Dissertação apresentada à Faculdade de \\ Medicina da Universidade de São Paulo para \\ obtenção do título de Mestre em Ciências \\ Programa de Ciências da Reabilitação \\ Orientadora: Profa. Dra. Renata Hydee Hasue \\ Villibor
}

(Versão corrigida. Resolução CoPGr 6018/11, de 1 de novembro de 2011. A versão original está disponível na Biblioteca da FMUSP)

São Paulo

2020 
Dados Internacionais de Catalogaçăo na Publicaçăo (CIP)

Preparada pela Biblioteca da

Faculdade de Medicina da Universidade de Sāo Paulo

Creproduçăo autorizada pelo autor

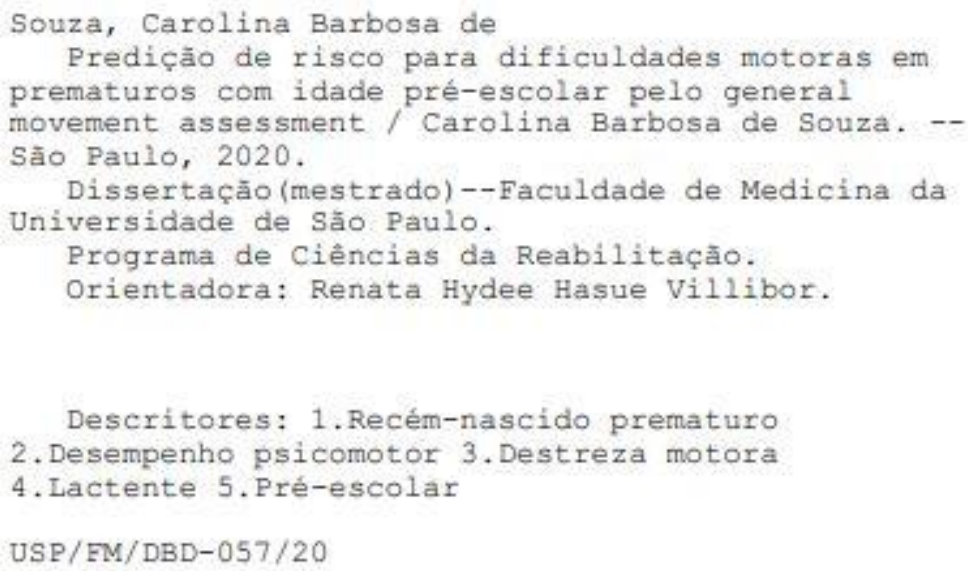

Responsável: Erinalva da Conceiçăo Batista, CRB-8 6755 


\title{
Dedicatória
}

Dedico este trabalho à todas as pessoas que contribuíram para o meu aprendizado e para o processo de construção desta pesquisa, resultado de muito esforço, empenho e dedicação:

A toda minha família, especialmente aos meus pais, Maria Eliza e José Domingos, e ao meu irmão, Gustavo pelo amor, paciência, incentivo e principalmente por acreditarem em mim.

\begin{abstract}
À minha orientadora, Profa. Dra. Renata Hydee Hasue, pela dedicação, incentivo e ensinamento durante esses anos.

À minha avó Geralda e minha tia Marisa, por sempre me apoiarem e participarem de cada conquista.
\end{abstract}

Ao meu avô, Onei Barbosa, que sempre torceu muito por mim em todos os momentos de minha vida.

Sei que continua na torcida onde estiver. 


\section{Agradecimentos}

Primeiramente aos meus pais Maria Eliza Barbosa de Souza e José Domingos de Souza por me proporcionarem e me guiarem por este caminho que escolhi para seguir a minha profissão, sempre com muito amor e paciência. Ao meu irmão, Gustavo Barbosa de Souza por ser o equilíbrio na família.

À minha orientadora, Profa. Dra. Renata Hydee Hasue, por toda a dedicação e doação durante a realização desta dissertação, desde o início em 2017 que me aceitou no Laboratório de Avaliação Neurofuncional.

À Dra. Silvia Maria Ibidi e toda equipe do Ambulatório de Prematuros do Hospital Universitário da Universidade de São Paulo - USP, pela parceria disponibilizando as crianças para a pesquisa.

Ao prof. Dr. Jorge Alberto de Oliveira por abrir as portas da Universidade de São Paulo - USP. Ao Grupo de Estudo de Atenção ao Desenvolvimento Infantil - GEADI, por todas as reuniões e discussões que foram fundamentais para a construção deste trabalho.

A todas as queridas amigas e colegas do Laboratório de Avaliação Neurofuncional pela ajuda no decorrer destes anos. A Liga de Fisioterapia e Neonatologia LIFINEOPED que fizeram essa jornada mais leve.

Em especial a minha amiga e companheira de Laboratório, por sempre me apoiar e me ensinar um caminho mais fácil dentro da pós-graduação, e por toda parceria que construímos nesses anos juntas, Dra. Carolina Aizawa.

Aos meus amigos de colégio e de vida que sempre compreenderam minha ausência durante todo esse processo, e estiveram ao meu lado.

À todas as crianças e famílias que se comprometeram e participaram deste trabalho sem eles, nada seria possível.

Aos professores da banca de qualificação pelas valiosas sugestões e contribuições que enriqueceram este trabalho. 
O presente trabalho ainda foi realizado com apoio da Coordenação de Aperfeiçoamento de Pessoal de Nível Superior - Brasil (CAPES) - Código de Financiamento 001.

Ao CNPQ (Conselho Nacional de Desenvolvimento Científico e Tecnológico), pela bolsa de estudos e auxílio financeiro que possibilitou dedicação exclusiva ao programa de pós-graduação. 
A ciência é uma grande coisa quando está a nossa disposição; no seu verdadeiro sentido, é uma das palavras mais formidáveis do mundo. 


\section{Normatização Adotada}

Esta dissertação está de acordo com as seguintes normas, em vigor no momento desta publicação:

Referências: adaptado de Internacional Committee of Medical Journals Editors (Vancouver).

Universidade de São Paulo. Faculdade de Medicina. Divisão de Biblioteca e Documentação. Guia de apresentação de dissertações, teses e monografias. Elaborado por Anneliese Carneiro da Cunha, Maria Julia de A.L. Freddi, Maria F. Crestana, Marinalva de Souza Aragão, Suely Campos Cardoso, Valéria Vilhena. 3a Ed. São Paulo: Serviços de Biblioteca e Documentação; 2011.

Abreviaturas dos títulos dos periódicos de acordo com List of Journals Indexed in Index Medicus. 


\section{SUMÁRIO}

\section{Lista de Abreviaturas e Siglas}

Lista de Figuras

Lista de Tabela

Resumo

$1 \quad$ Abstract

2 INTRODUÇÃO.

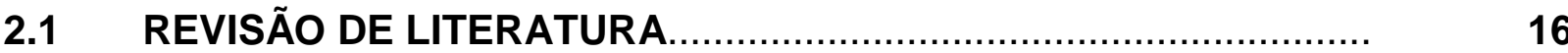

$2.2 \quad$ Desenvolvimento motor e fatores de risco...................................... 16

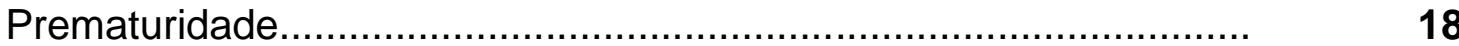

2.3 Transtorno do Desenvolvimento da Coordenação............................ 21

$2.4 \quad$ Avaliação motora........................................................................ $\quad 25$

2.4.1 Movement Assessment Battery for Children (MABC-2) ..................... 25

2.4.2 General Movement Assessment (GMA)................................... 27

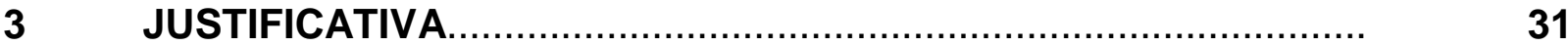

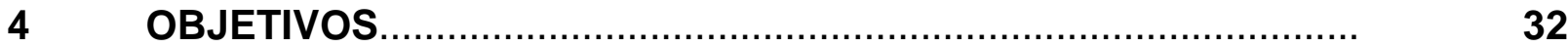

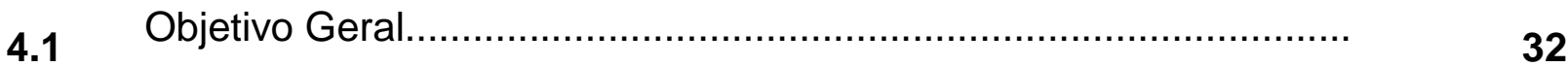

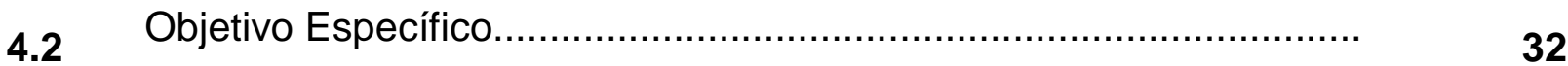

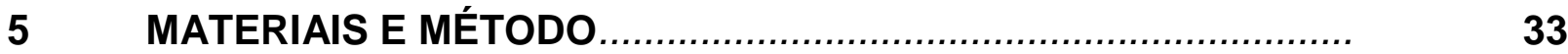

5.1 Tipo de Estudo................................................................. 33

$5.2 \quad$ Local de Estudo....................................................................

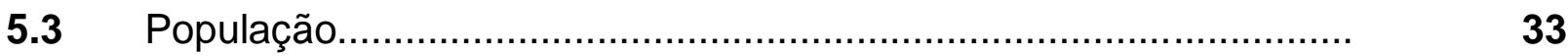

5.3.1 Amostra..............................................................................

5.3.2 Critérios de Inclusão.......................................................... $\quad 34$

5.3.3 Critérios de Exclusão........................................................... $\quad 34$ 


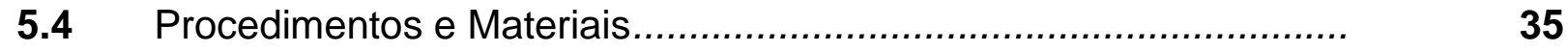

5.4.1 Avaliação pelo General Movement Assessment............................ 36

5.4.2 Avaliação motora - Movement Assessment Battery for Children 2... $\quad 37$

5.5 Análise Estatística............................................................... $\quad 38$

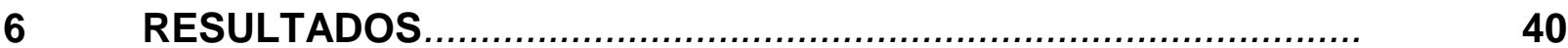

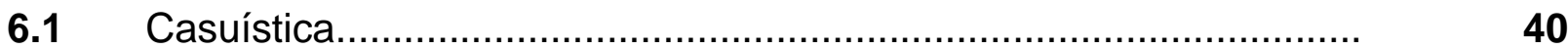

6.2 Resultados descritivos do GMs e do MABC-2 ............................... 41

6.3 Validade preditiva do GMA para o desfecho motor pelo MABC-2...... 43

6.4 Influência dos fatores clínicos e sociodemográficos no desempenho motor ....................................................................... 46

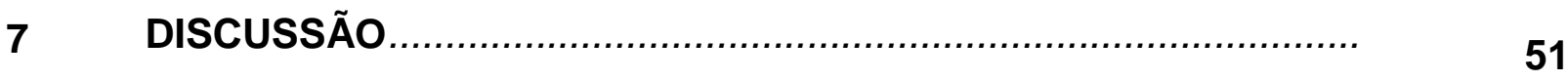

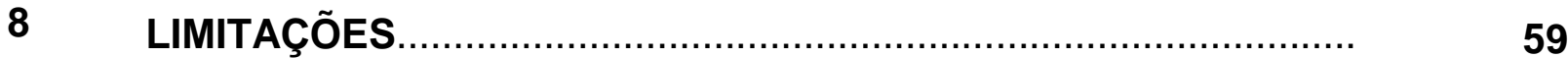

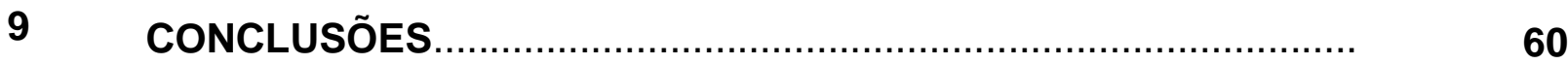

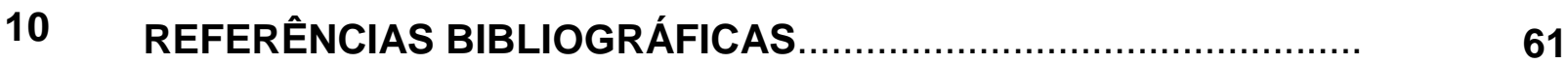

11 ANEXOS $\quad 74$

$\begin{array}{ll}\text { Anexo } 1 & 74\end{array}$

$\begin{array}{ll}\text { Anexo } 2 & 77\end{array}$

$\begin{array}{ll}\text { Anexo } 3 & 78\end{array}$

$\begin{array}{ll}\text { Anexo } 4 & 79\end{array}$

$\begin{array}{ll}\text { Anexo } 5 & 81\end{array}$ 


\section{Lista de Abreviaturas e Siglas}

TDC Transtorno do Desenvolvimento da Coordenação

SNC Sistema Nervoso Central

GMA General Movement Assessment

PC

Paralisia Cerebral

OMS Organização Mundial de Saúde

APA American Psychological Association

DSM-V Diagnostic and Statistical Manual of Mental Disorders - Fifth Edition

IG Idade Gestacional

GMs General Movements

MABC-2 Movement Assessment Battery for Children - Second Edition

SINASC Sistema de Informações sobre Nascidos Vivos

WM Writhing movements

FM Fidgety movements

HU Hospital Universitário

USP Universidade de São Paulo

BSID Bayley Scales of Infant Development

RN Recém Nascido

HIV Vírus da Imunodeficiência Humana 


\section{Lista de Tabelas}

Tabela 1 Características clínicas da amostra...................................... 41

Tabela 2 Avaliação pelo GMA........................................................ 42

Tabela 3 Avaliação pelo MABC-2 ..................................... 43

Tabela 4 Desfecho motor do GMA pelo MABC-2 ….............................. 44

Tabela 5 Valores preditivos do GMA para o desfecho motor pelo MABC-2

Tabela 6 Caracterização entre grupos em relação às variáveis clínicas. 47

Tabela 7 Resultados do MABC-2 em relação as variáveis clínicas e sociodemográficas 


\section{Lista de Figuras}

Figura 1 Critérios diagnósticos do TDC segundo DSM-5...................... 24

Figura 2 Fluxograma do processo de amostragem dos participantes........ 40

Figura 3 Comparação da classificação na fase dos writhing movements pelo GMA com o Percentil total pela MABC-2 ...................... 45

Figura 4 Comparação de todos os domínios da MABC-2 em relação aos resultados obtidos na fase dos fidgety movements pelo GMA..... 46 


\section{Resumo}

Souza CB. Predição de risco para dificuldades motoras em prematuros em idade pré-escolar pelo general movement assessment [dissertação]. São Paulo: Faculdade de Medicina, Universidade de São Paulo; 2020.

Introdução: Os avanços científicos e tecnológicos na neonatologia resultaram em aumento na sobrevivência de prematuros, e, consequentemente, no maior número de crianças com alterações do desenvolvimento. Frequentemente, dificuldades motoras como prejuízos no equilíbrio e na coordenação só são observadas na idade escolar. A detecção precoce e eficaz é fundamental para a intervenção e minimização dos danos funcionais, psicológicos e emocionais que impactam diretamente na qualidade de vida dessas crianças. Objetivos: Identificar se o repertório motor nos primeiros meses de vida de prematuros está relacionado com as dificuldades motoras observadas em idade pré-escolar e correlacionar com fatores clínicos e sociodemográficos. Método: Estudo longitudinal prospectivo com prematuros avaliados pelo General Movement Assessment (GMA) até os 4 meses de idade corrigida e reavaliados pelo Movement Assessment Battery for Children-2 (MABC-2) entre os 3 e 5 anos de idade. A One Way Anova foi usada para identificar diferença entre grupos e o teste de Fisher Exact para associação entre avaliações e fatores clínicos e sociodemográficos. Resultados: Dos 23 prematuros avaliados (65.3\% masculinos, idade gestacional média de 31 semanas) 56.3\% apresentaram writhing movements anormais, sendo $30.4 \%$ com dificuldades motoras pela MABC-2. Os fidgety movements estavam anormais em $26.1 \%$, e, destes, $21.7 \%$ manifestaram dificuldades motoras. A melhor destreza manual foi associada com maior escolaridade materna $(p=0.035)$. As crianças que fizeram fisioterapia nos dois primeiros anos de idade apresentaram mais dificuldades motoras. Conclusão: Nossos achados sugerem que a escolaridade materna parece ser um fator protetivo para a destreza manual. Além disso, as crianças prematuras devem ser acompanhadas com abordagens motoras ininterruptas até a idade escolar, a fim de prevenir prejuízos no desempenho motor.

Descritores: Recém-nascido prematuro; Desempenho psicomotor; Destreza motora; Lactente; Pré-escolar. 


\begin{abstract}
Souza CB. Prediction of risk for motor difficulties in premature infants at preschool age by general movements assessment [dissertation]. São Paulo: "Faculdade de Medicina, Universidade de São Paulo“; 2020.

Introduction: Scientific and technological advances in neonatology have resulted in increased survival of preterm infants, and, consequently, in a greater number of children with developmental disorders. Motor difficulties such as impaired balance and coordination are often only observed at school age. Early and effective detection is essential for intervention and minimization of functional, psychological and emotional impairments that directly impact the quality of life of these children. Objectives: Identify whether motor repertoire in the first months of life of preterm infants is related to the motor difficulties observed at preschool age and to correlate with clinical and sociodemographic factors. Method: Prospective longitudinal study with preterm infants born between 2013 and 2015, assessed by the General Movement Assessment (GMA) up to 4 months of corrected age and reassessed by the Movement Assessment Battery for Children-2 (MABC-2) between 3 and 5 years of age. One Way Anova was used to identify differences between groups and the Fisher Exact test for the association between assessments and clinical and sociodemographic factors. Results: Of the 23 preterm infants assessed $(65.3 \%$ male, mean gestational age of 31 weeks) $56.3 \%$ had abnormal writhing movements, and $30.4 \%$ had also motor difficulties by MABC-2. Fidgety movements were abnormal in $26.1 \%$, and $21.7 \%$ had concomitant motor difficulties. Lower motor difficulties in manual dexterity are associated with higher degree of maternal education $(p=0.035)$, and children who underwent physical therapy in the first two years of age had more motor difficulties. Conclusion: Our findings suggest that maternal education seems to be a protective factor for manual dexterity. In addition, premature children should be accompanied with uninterrupted motor approaches until school age, in order to minimize impairment in motor performance.
\end{abstract}

Descriptors: Infant, premature; Physomotor, performance; Motor skills; Infant; Child, preschool. 


\section{INTRODUÇÃO}

Dificuldades motoras na infância podem interferir significativamente no desempenho das atividades cotidianas da criança, como brincadeiras, tarefas de autocuidado e atividades escolares, gerando muitas vezes incapacidade da criança desempenhar suas atividades de vida diária de forma adequada (Santos et al., 2004; Petersen e Oliveira., 2004).

Atividades de autocuidado como vestir-se e utilizar talheres, tarefas acadêmicas como copiar, organizar a mesa de trabalho, atividades de ginástica, esportes e jogos infantis podem tornar-se desafios, e mesmo que nem todas as crianças apresentem as mesmas dificuldades, seu desempenho motor será mais lento, com menor precisão, em relação aos seus pares (Missuna et al., 2008). Essas dificuldades podem continuar até a adolescência e idade adulta e não são justificadas por desordem neurológica ou deficiência intelectual, afetando de forma significativa a vida diária e a interação social (Zwicker et al., 2013; Zwicker et al., 2012).

A condição que caracteriza essas dificuldades motoras, recebeu diversos nomes ao longo dos anos, como disfunção cerebral mínima, dispraxia, síndrome da criança desajeitada, entre outros (Polatjko et al., 1995). Recentemente foi nomeada com o termo Transtorno do Desenvolvimento da Coordenação (TDC) e foi introduzido no Diagnostic and Statistical Manual III - DSM-III-TR, em 1994, no entanto, a Associação Americana de Psiquiatria (APA, 1994), com a publicação da quarta edição do Manual Estatístico de Diagnósticos de Distúrbios Mentais (DSMIV), reafirmou o termo Transtorno do Desenvolvimento da Coordenação (Developmental Coordination Disorder - DCD) e apresentou critérios específicos 
para o diagnóstico. Recentemente, em 2013, a APA lançou o DSM-5 que apresentou novas características para os critérios diagnósticos do TDC.

O TDC é definido como um comprometimento no aprendizado e execução de habilidades motoras, abaixo do esperado para idade cronológica e a oportunidade de aquisição e uso das habilidades. Além disso, a dificuldade motora interfere significativamente e persistentemente no desempenho das atividades de vida diária, produtividade acadêmica e de lazer. Os sinais do TDC aparecem no início do desenvolvimento infantil, e essa dificuldade não pode ser atribuída a uma deficiência intelectual, deficiência visual ou condição neurológica (APA, 2013).

O impacto do TDC na vida da criança ocorre em diversos aspectos como social, no desempenho escolar e na participação em atividades motoras típicas para a idade, tornando-se necessária a identificação precoce destas dificuldades, possibilitando intervenções preventivas para evitar efeitos secundários do transtorno, como o isolamento social e o baixo desempenho escolar (Cantel et al., 2003; Miller et al., 2001; Missuna et al., 2011; Prado, 2007; Tseng et al., 2010). É essencial a avaliação criteriosa da criança para a identificação dos sinais de alterações no desempenho motor. Mesmo sem a possibilidade de fazer diagnóstico definitivo principalmente em idade pré-escolar, é importante contar com recursos que podem beneficiar a instrução mais dirigida ou programas individualizados de atividades motoras (Okuda, 2015).

A infância é a etapa mais importante durante o processo de maturidade até a vida adulta, por isso é necessário garantir que esse período traga condições propícias e pertinentes para a sua evolução e para o seu desenvolvimento motor (Moreira, 2000). 


\section{REVISÃO DE LITERATURA}

\subsection{Desenvolvimento motor e fatores de risco}

O desenvolvimento motor é considerado como um processo sequencial contínuo e não-linear, relacionado com a idade cronológica, pelo qual o ser humano adquire uma enorme variedade de habilidades motoras (Willrich et al., 2009). É um processo que se inicia na vida intrauterina e envolve a maturação do sistema nervoso central (SNC), a interação com o meio ambiente e os estímulos dados à criança durante o seu desenvolvimento, progredindo de padrões de movimentos simples e desorganizados para movimentos mais complexos, voluntários e altamente organizados (Diament e Cypel, 2005; Moraes, 2010). Esses padrões de movimentos são caracterizados por duas mudanças fundamentais: o aumento da diversificação e o aumento da complexidade (Clark, 2007).

Diversos pesquisadores da área interpretam o processo do desenvolvimento motor como um fenômeno determinado por múltiplos fatores no qual as contínuas interações entre o indivíduo, o ambiente no qual ele está inserido e as próprias características dos movimentos que ele executa formam a base do processo de integração entre suas funções sensoriais e motoras, que contribuem para o desenvolvimento de novas habilidades motoras, pela formação de novas redes neurais e do estabelecimento e reorganização de sinapses (Magill, 2011; Braga, 2005). Dessa forma as mudanças desenvolvimentais podem ser desencadeadas e influenciadas pela biologia do indivíduo, pelas condições ambientais e socioeconômicas e também pelas necessidades da tarefa (Gallahue et al., 2013).

Diversas condições biológicas e ambientais interferem diretamente na primeira infância e podem determinar a susceptibilidade ao atraso no 
desenvolvimento neuropsicomotor (Ribeiro et al., 2014). Os fatores de risco biológico para o desenvolvimento infantil são: prematuridade, asfixia perinatal, hemorragia periventricular, displasia broncopulmonar, distúrbios bioquímicos do sangue (hipoglicemia, policitemia e hiperbilirrubinemia), malformações congênitas, infecções congênitas ou perinatais (vírus Zika, Toxoplasmose, Sífilis, Rubéola, Herpes, Vírus da Imunodeficiência Humana - HIV, Citomegalovírus), restrição de crescimento intrauterino e mães usuárias de drogas (Resegue et al., 2007). Os principais fatores de risco ambientais incluem condições de moradia, escolaridade dos pais e/ou cuidadores, dinâmica familiar, estado nutricional e poder aquisitivo familiar. Estes podem podem limitar o fornecimento de recursos materiais e sociais para as crianças, o que, por sua vez, afeta seu desenvolvimento geral (Piccolo et al., 2012; Ermisch et al., 2013; Pedraza et al., 2014).

A caracterização do desenvolvimento motor na infância por meio da aquisição e combinação de diversas habilidades motoras, denominadas básicas, possibilita à criança um domínio do seu corpo em diferentes posturas (estáticas e dinâmicas) para locomover-se pelo meio ambiente e para realizar diferentes atividades de manipulação de objetos, brinquedos e instrumentos (Santos et al., 2004). Embora o desenvolvimento motor implique mudança, a representação mais comum desse processo enfoca a estabilidade do comportamento, por exemplo, em sequências de desenvolvimento, podem sofrer diversas alterações com a idade e são inerentes às diferenças sociais, cognitivas e emocionais (Manoel, 1994;Payne e Isaacs, 2007).

Os parâmetros de normalidade da sequência do desenvolvimento motor servem como padrões típicos do desenvolvimento infantil, representando a idade média em que a criança é capaz de realizar habilidades motoras básicas, que são requisitadas no seu cotidiano (Tani, 1987; Santos et al., 2004). A identificação 
dessas sequências pode ser utilizada como referência para a identificação e avaliação de estados do desenvolvimento motor dos indivíduos ao longo do ciclo da vida (Tani et al., 2010). Portanto, a avaliação motora é importante para o conhecimento de informações relacionadas ao desenvolvimento motor da criança típico ou atípico (Medina-Papst e Marques, 2010).

\subsection{Prematuridade}

De acordo com a Organização Mundial de Saúde (OMS), o termo prematuridade é definido quando o parto ocorre com idade gestacional inferior a 37 semanas, ou seja, 36 semanas e 6 dias. Em relação à idade gestacional (IG), ainda podem ser classificados como: prematuridade moderada (nascimento ocorre entre a $32^{\mathrm{a}}$ semana de gestação e a $36^{\mathrm{a}}$ semana e 6 dias), prematuridade acentuada (nascimento entre a $28^{\mathrm{a}}$ semana de gestação e a $31^{\mathrm{a}}$ semana e 6 dias) e prematuridade extrema (inferior a 28 semanas de idade gestacional) (OMS - 1977). Os recém-nascidos prematuros também podem ser classificados quanto ao peso ao nascer: denomina-se prematuro baixo peso aquele nascido com menos de $2 \mathrm{~kg}$, muito baixo peso com menos de $1,5 \mathrm{~kg}$ e extremo baixo peso com menos de $1 \mathrm{~kg}$ (Ministério da Saúde, 2017).

Anualmente, nascem aproximadamente 15 milhões de recém nascidos prematuros no mundo, uma incidência de um para cada dez nascimentos. A prematuridade é a segunda maior causa de mortalidade infantil (Ministério da Saúde 2017; OMS 2018). De acordo com os dados do último Sinasc 2017, o Brasil teve 
319.312 casos de partos prematuros por ano, sendo o estado de São Paulo o maior com número de partos prematuros do país, representando $22 \%$ dos casos.

Os avanços científicos que ocorreram na área de neonatologia nas últimas décadas, especialmente a implantação da Unidade de Terapia Intensiva (UTI) neonatal, contribuíram para o aumento das taxas de sobrevida dos lactentes prematuros (Rugolo, 2005; Santos et al., 2008). Atualmente, recém-nascidos com idades gestacionais cada vez menores recebem alta das unidades de cuidados neonatais em condições aparentemente satisfatórias (Resegue, 2007).

O parto prematuro traz repercussões importantes para área de saúde pública, uma vez que é considerado de risco para o desenvolvimento infantil saudável, pois as implicações para eles adquirirem morbidades neurológicas, alterações de desenvolvimento e dificuldade de aprendizagem são de duas a três vezes maiores do que crianças nascidas a termo. Dentre as principais alterações estão: transtornos de coordenação, atraso no crescimento pôndero-estatural, deficiência cognitiva, perda auditiva, comprometimentos visuais, alterações de linguagem, problemas cardiovasculares, problemas respiratórios, déficits de atenção, hiperatividade, dificuldade de equilíbrio e, em casos mais graves, a paralisia cerebral (PC) (Rogers \& Hintz 2016; Hack, 2006; Allen, 2008).

A prematuridade, o muito baixo peso ao nascer e as complicações moderadas ou severas neonatais podem ocasionar outros fatores biológicos adversos, tais como hospitalização repetida ou prolongada, relacionada a necessidade de suporte ventilatório, displasia broncopulmonar, hemorragia intracraniana e icterícia neonatal. Esse conjunto de fatores contribuem para aumentar o risco de prejuízos para o desenvolvimento (Linhares, 2003; Sullivan e McGrath, 2003). 
Uma parcela pequena de crianças nascidas de parto prematuro são diagnosticadas com PC, cerca de 10 a 15\% (Orton et al., 2009). Entretanto, existem outras alterações neuropsicomotoras detectadas em idade escolar e compreendem alterações de coordenação motora, déficit de equilíbrio, dificuldade de aprendizagem e de linguagem (Marlow et al., 2007; Bhutta et al., 2002). Estima-se que aproximadamente $50 \%$ das crianças que nascem de parto prematuro apresentam algum distúrbio do desenvolvimento (Greene et al., 2013). É comum correlacionar a prematuridade com a PC, no entanto estudos de acompanhamento de longo prazo encontraram taxas mais elevadas para problemas motores leves: 25 a $50 \%$ de crianças nascidas pré-termo (Fawke, 2007). As crianças nascidas prematuras são mais propensas a apresentar dificuldades motoras e cognitivas que seus pares nascidos a termo. Diversos fatores parecem contribuir negativamente para potencializar os riscos biológicos no desfecho do desenvolvimento dessas crianças. Como a maioria delas apresenta desenvolvimento aparentemente normal, suas dificuldades, muitas vezes, só são mais evidentes na idade escolar, quando as exigências motoras e cognitivas são maiores (Maggi, 2012).

As crianças prematuras que apresentam dificuldades discretas em habilidades motoras, de comportamento, no desempenho escolar e na linguagem, poderão também apresentar limitações e restrições sociais ao longo da vida e muitas vezes não receberão um diagnóstico específico e acompanhamento adequado (Rademaker et al., 2004). Cerca de 30 a $42 \%$ das crianças nascidas de parto prematuro apresentam alterações motoras características do TDC (FoulderHughes e Cooke, 2003; Goyen e Lui, 2009). Em crianças que nascem com idade gestacional $\leq 32$ semanas, as chances de desenvolver o TDC aumentam em oito vezes (Edwards, 2011). 


\subsection{Transtorno do Desenvolvimento da Coordenação (TDC)}

O Transtorno do Desenvolvimento da Coordenação (TDC) é caracterizado pelo atraso no desenvolvimento da coordenação motora (Sugden, 2006). Crianças com TDC apresentam baixo desempenho motor para a idade cronológica, que interfere significativa e persistentemente no desempenho das atividades de vida diária (AVDs), com impacto sobre a produtividade escolar e de lazer (APA, 2013). Essas crianças desenvolvem maior nível de introversão, alto nível de ansiedade e baixos níveis de aptidão e atividade física (Schoemaker e Kalverboer, 1994; Nascimento et al., 2013).

Vaivre-Douret (2014) relatou que o TDC além de gerar atrasos no desenvolvimento motor, acarreta em: pobre coordenação sensório-motora, equilíbrio deficitário, dificuldades com novas habilidades, alteração no planejamento do movimento, desordens na sequência de movimento (timing) e também alguns déficits no processo de informação visual e espacial.

Estudos indicam que o TDC afeta $5 \%$ a $20 \%$ da população infantil, sendo que $5 \%$ a $6 \%$ são estimativas mais reportadas na literatura (APA, 2013). É mais comum nos meninos em proporções que variam de 2 a 7 meninos para 1 menina (Blank et al., 2012).

Apesar dessa prevalência classificá-lo como um dos transtornos mais frequentes em crianças (Wann, 2007), muitos profissionais das áreas da saúde e educação que trabalham com crianças subestimam o TDC e acreditam que o processo desenvolvimental resolverá este atraso ao longo do tempo, mesmo que 
estudos apontem que o TDC persiste durante toda a vida (Blank et al., 2012; Cantell, 1994; Geuze e Borger, 1993; Losse et al., 1991; Barnhart et al., 2003).

A etiologia do TDC ainda não é bem definida, no entanto acredita-se que seja multifatorial. Alguns estudos apontam a correlação com anormalidades observadas em exames de neuroimagem e à coordenação motora, mas os dados não são conclusivos (Barnett et al., 2002; Zwicker et al., 2013). Outros autores discutem a relação entre prejuízos cerebrais, complicações perinatais, prematuridade, baixo peso ao nascimento e fatores familiares (Fawke, 2007; Jongmans et al., 1998; Magalhães et al., 2009). Sabe-se também que os problemas motores não constituem uma condição isolada, sendo freqüente a associação com as dificuldades de aprendizagem e o transtorno do déficit de atenção e hiperatividade (Kaplan et al., 2006; Dewey et al., 2002; Green e Baird, 2005).

Os baixos níveis socioeconômicos, somados aos fatores biológicos, podem aumentar o risco de pobre desempenho motor. Nos países em desenvolvimento, os estímulos e as oportunidades oferecidas às crianças podem contribuir para a alta prevalência dos problemas motores. Neste sentido, é possível que as crianças brasileiras tenham menores estímulos motores e talvez não tenham a mesma qualidade e quantidade de experiência motora que crianças de países desenvolvidos, comprometendo a coordenação motora (Valentini et al., 2015; Santos et al., 2015).

A diferença na demanda da cultura corporal do movimento em relação a diferentes nacionalidades foi um dos motivos pelo qual o atual sistema de identificação do TDC, circunscrito no Diagnostic and Statistical Manual of Mental Disorders - Fifth Edition (DSM-5), em concordância aos resultados de estudos dos 
últimos anos, estabeleceu quatro critérios $(A, B, C$ e D) para diagnosticar o TDC de forma mais precisa em comparação com a última versão do manual (Figura 1).

Os critérios A e B sugerem observar se o aprendizado e execução de habilidades motoras coordenadas estão adequados para a idade cronológica, em relação à possibilidade de "oportunidade para a aprendizagem e uso dessas habilidades". Deve-se observar se o desempenho da criança na habilidade motora interfere significativa e persistentemente nas AVD's com impactos sobre a produtividade escolar/acadêmica, atividades pré-profissionais e profissionais, de lazer e diversão (APA, 2013).

De acordo com o critério $C$, a manifestação de sintomas do TDC deve estar presente desde o período inicial do desenvolvimento, expressando-se por exemplo no tempo de aprendizagem de novas habilidades (APA, 2013). Já no critério D recomenda-se que o diagnóstico do TDC deve ser feito se os atrasos nas habilidades motoras não são mais bem explicados por deficiência visual e não são atribuíveis a condições neurológicas que afetem o movimento (por exemplo, paralisia cerebral, distrofia muscular e doença degenerativa). Nesse caso, são recomendados exames oftalmológicos e neurológicos (APA, 2013). 


\begin{tabular}{|c|c|}
\hline Critérios & Características \\
\hline A & $\begin{array}{l}\text { A aprendizagem e execução de habilidades motoras estão substancialmente abaixo do esperado } \\
\text { dadas a idade cronológica e a oportunidade para a aprendizagem e uso das habilidades. } \\
\text { Dificuldades se manifestam por desajeitamento (clumsiness) (por exemplo, derrubar ou bater em } \\
\text { objetos), bem como lentidão e imprecisão no desempenho de habilidades motoras, como pegar um } \\
\text { objeto, usar uma tesoura ou talheres, na escrita, andar de bicicleta ou participar de um esporte. }\end{array}$ \\
\hline B & $\begin{array}{l}0 \text { déficit nas habilidades motoras descritas no critério } \mathrm{A} \text { interferem significativamente } \\
\text { persistentemente nas atividades da vida diária apropriadas para a idade cronológica (por exemplo, } \\
\text { autocuidado ou automanutenção) com impactos no rendimento acadêmico / escolar, nas atividades } \\
\text { pré-profissional e profissional, lazer e diversão. }\end{array}$ \\
\hline $\mathrm{C}$ & 0 início dos sintomas deve estar presente desde o período inicial do desenvolvimento. \\
\hline D & $\begin{array}{l}\text { Os déficits de habilidades motoras não são explicados por deficiência intelectual (transtorno do } \\
\text { desenvolvimento intelectual) ou deficiência visual e não é atribuivel a uma condição neurológica } \\
\text { que afeta o movimento (por exemplo, paralisia cerebral, distrofia muscular ou doença } \\
\text { degenerativa) }\end{array}$ \\
\hline
\end{tabular}

Fonte: APA (2013).

Figura 1: Critérios diagnósticos do TDC segundo DSM-5

O diagnóstico do TDC, deve ser feito por uma avaliação integral que inclua o conhecimento sobre a história do indivíduo, exame físico, relatório da escola ou do local de trabalho e avaliação individual, usando um teste padronizado e culturalmente apropriado. A abordagem ideal envolve um processo multidisciplinar que inclui a criança, a família, e os profissionais da saúde e da educação (APA, 2013). 


\subsection{Avaliação motora}

\subsubsection{Motor Movement Assessment Battery for Children (MABC-2)}

Para a identificação e avaliação do desempenho motor de crianças com TDC, o Motor Movement Assessment Battery for Children (MABC-2) é o teste mais utilizado. Foi desenvolvido no Reino Unido para identificar dificuldades motoras em crianças com idade entre 3-16 anos por meio das seguintes habilidades: destreza manual, habilidades com bola e equilíbrio estático e dinâmico, organizado de acordo com cada faixa etária (Henderson et al., 2007). O MABC-2 é uma ferramenta amplamente utilizada tanto em estudos nacionais (Miranda et al., 2007; Silva 2013) quanto em estudos internacionais. Foi realizada a tradução, a confiabilidade e a validade da segunda versão do MABC para crianças brasileiras (Valentini et al., 2013).

O MABC-2 é um protocolo de teste motor desenvolvido para a avaliação de crianças com TDC e outros problemas motores. É constituída por uma bateria de movimento, uma lista de controle, e um manual (Henderson et al., 2007). O MABC-2 está organizado em três bandas específicas por idade:

Banda 1: 3-6 anos;

Banda 2: 7-10 anos;

Banda 3: 11-16 anos.

Dentro de cada banda as atividades motoras se diferem em dificuldade de acordo com a idade. O teste refere-se a uma série de tarefas (destreza manual, habilidades com bola e equilíbrio estático e dinâmico), atribuído a um valor como 
número de tentativas, erros e acertos, ou o tempo gasto para executar as tarefas (Henderson et al., 2007).

O teste categoriza as crianças de acordo com o seu nível de dificuldade motora. A pontuação varia de 1 a 19, e para cada valor, existe uma percentagem correspondente, que pode variar de $0,1 \%$ a $99,9 \%$. Uma pontuação igual ou inferior ao quinto percentil é o ponto de corte para dificuldades motoras, como TDC; valores entre o sexto e décimo quinto percentil são considerados para indicar o risco para o desenvolvimento de dificuldades motoras; desempenho igual ou superior ao percentil dezesseis indica que não há dificuldades (Henderson et al., 2007).

O TDC pode se manifestar no período inicial do desenvolvimento, porém a condição muitas vezes não é identificada antes da idade escolar (Missiuna et al., 2007). Vários estudos afirmaram que o MABC-2 é uma ferramenta confiável e válida para avaliar distúrbios do movimento na banda 1 (idade: 3-6 anos) (Henderson et al., 2007; Ellinoudis et al., 2011).

Segundo, Smits-Engelsman et al., (2011) o MABC-2 tem excelente confiabilidade teste-reteste em crianças de três anos de idade com desenvolvimento motor típico. No entanto, há limitações sobre a validade preditiva do MABC-2 nas crianças prematuras na mesma faixa etária.

Pesquisas têm evidenciado que a identificação precoce, avaliação e intervenção podem auxiliar minimizando o impacto subjacente do TDC ao longo da vida, como problemas de saúde, sociais e psicológicos, que afetam a autoestima, o autoconceito e a percepção de competência (Cousins; Smyth, 2003; Cantell et al., 2003; Kirby et al., 2014; Schoemaker et al., 2006). 


\subsubsection{General Movement Assessment (GMA)}

O método que oferece melhor predição de dificuldades motoras, como é o caso da PC, nos primeiros meses de vida de uma criança prematura consiste no General Movement Assessment - GMA (Noble e Boyd, 2012).

Os General Movements (GMs) são parte do repertório motor espontâneo infantil, desde a vida fetal até mais ou menos quatro meses pós-termo de idade corrigida (Einspieler e Prechtl, 2005). A análise qualitativa dos GMs é caracterizada pela percepção da Gestalt: complexidade, variabilidade e fluência do movimento; definidos como movimentos endógenos e ativos que refletem a maturação do sistema nervoso central (SNC).

Os GMs são movimentos espontâneos, com um repertório rico e complexo, com rotações ao longo do eixo dos membros e ligeiras mudanças na direção do movimento que os tornam fluentes e elegantes, e criam a impressão de complexidade e variabilidade. Envolvem o corpo inteiro em uma sequência variável de movimentos dos membros superiores, membros inferiores, pescoço e tronco. Eles aumentam e diminuem aos poucos em vigor, intensidade e velocidade e têm início e um fim graduais (Einspieler e Prechtl, 2005; Groen et al., 2005; ZahedCheikh et al., 2011). Se o sistema nervoso da criança estiver comprometido, os GMs perdem seu caráter complexo e variável e se tornam monótono e pobre (Einspieler e Prechtl, 2005).

O GMA é, dentre todas as avaliações aplicadas nos primeiros meses de vida, a de maior valor preditivo para riscos de alterações no desenvolvimento e desfechos neurológicos adversos (Noble e Boyd, 2012). Estudos relatam altos valores de 
sensibilidade (95\%) e especificidade (96\%) para o GMA na predição do desfecho neurológico de lactentes prematuros ou a termos de risco. Além disso, esses valores foram superiores ao de exame de imagem de ultra-som (US) de crânio (Prechtl et al., 1997).

Ao longo dos anos, a análise aprofundada dos GMs permitiu a identificação de alguns padrões específicos de movimentos. Esses padrões são caracterizados em dois tipos, de acordo com a idade avaliada: os writhing movements e os fidgety movements. Os writhing movements (WMs) estão presentes desde o período termo até aproximadamente dois meses pós-termo, são caracterizados pelo movimento de todo o corpo e se manifestam em uma sequência variável de movimentos de pescoço, braço, tronco e pernas. Eles iniciam e terminam gradualmente, variando em intensidade e velocidade. Rotações e variações na direção fazem com que sejam complexos, porém suaves. Os WMs normais apresentam três características principais: variabilidade, complexidade e fluência. Eles se dissipam progressivamente, sendo substituídos pelos fidgety movements (FMs), caracterizados por movimentos de pequena amplitude, de velocidade moderada e aceleração variável de pescoço, tronco e membros em todas as direções. São contínuos no recém-nascido acordado e podem ser observados na 6a semana póstermo, mas geralmente ocorrem em torno da 9a semana, e permanecem até a 20a ou até um pouco mais tarde (Prechtl et al., 1997). A persistência de movimentação anormal até o período dos FM é preditiva de futuros distúrbios neurológicos. Ainda, a ausência desses movimentos permite a seleção de pacientes em risco de desenvolver PC (Prechtl, 2001; Einspièler e Prechtl, 2005).

Durante o período pré-termo e dos WMs, os movimentos generalizados considerados como anormais, apresentam uma redução da complexibilidade, 
variabilidade e fluência, assim classificados com repertório pobre, cramped synchronized e caótico (Pretchl et al., 1997). Os movimentos de repertório pobre apresentam a redução das características dos WMs, e podem apresentar componentes de rigidez ou hipocinesia. Esse tipo de alteração é inespecífica e pouco preditiva para o desenvolvimento motor, podendo ser encontrado em crianças que poderão desenvolver a PC (Pretchl et al., 1997), distúrbios do espectro autista (Einspieler, 2014), e também em crianças com desenvolvimento normal. Os GMs anormais, classificados como cramped synchronized, são caracterizados por rigidez, falta de fluência e elegância no movimento, contração e relaxamento simultâneo dos músculos. Possuem alto valor preditivo (98\%) para o diagnóstico de PC e quanto mais precoce esses movimentos aparecem, mais grave é o prejuízo funcional (Ferrari, 2002). Já os GMs caóticos são caracterizados por movimentos desorganizados, com velocidade e amplitude alta. Esses movimentos são raros de ser encontrados, aparecem na fase de termo e frequentemente evoluem para cramped synchronized (Einspieler, 2015).

Palmer (2004) mostrou que a movimentação espontânea anormal na criança entre a 16a e 20a semanas de vida, ou antes, reflete limitações funcionais precoces nos primeiros meses de vida e tem sido demonstrada preditora de PC. A avaliação da qualidade dos GMs, em particular a complexidade e a variabilidade entre dois e quatro meses pós-termo, pode não apenas ser usada como instrumento preditor de PC, mas pode ser aplicada como ferramenta para detectar crianças predispostas a desenvolver distúrbios neurológicos menores, incapacidade para atividades de manipulação fina e problemas de coordenação (Groen et al., 2005). Um estudo com crianças com idade escolar de 9 a 12 anos que nasceram de parto prematuro, mostra a correlação de GMs alterados em idades de 2 e 4 meses pós-termo, 
apresenta alto valor preditivo para PC, com também para disfunções neurológicas leves (Groen, 2005).

O valor preditivo dos GMs para detecção precoce de PC já está claro na literatura, e alguns estudos estão começando a investigar se esse valor se aplica para outras alterações motoras, como é o caso do TDC. Foi observado que em $64 \%$ das crianças com FM anormal ou ausente irão apresentar disfunções neurológicas menores na faixa etária de 7 a 11 anos (Janneke et al., 2008). Entretanto, neste estudo a amostra não excluiu as crianças com PC ou outras anormalidades, portanto, não sabemos a estimativa da prevalência específica do TDC.

Esta relação preditiva dos GMs para disfunções neurológicas mínimas também foi verificada numa faixa etária menor, em crianças com idade de 2 a 4 anos. Foram ultilzadas as escalas Bayley Scales of Infant Development (BSID) para cognição e linguagem, e o MABC-2 para avaliação motora fina e grossa. Entretanto, neste estudo as crianças com PC e outras doenças neurológicas também não foram excluídas (Spittle et al., 2013)

Em um estudo recente de Spittle et al., (2017) foi realizada avaliação por meio do GMA em 97 lactentes entre 1 e 3 meses, nascidos de parto prematuro. Essas crianças foram acompanhadas até 4 anos de idade e foi realizado o MABC-2. Foi observado alta predição na avaliação pelos GMs com 3 meses para as alterações motoras com 4 anos; porém nesse estudo as crianças com diagnóstico de paralisia cerebral também não foram excluídas da pesquisa. Desta forma, as alterações motoras encontradas não podem ser caracterizadas como TDC. 


\section{JUSTIFICATIVA}

A infância, principalmente no início do processo de escolarização, representa uma fase importante para aquisição de habilidades motoras, possibilitando à criança um amplo domínio do seu corpo em diferentes atividades (Biscegli et al., 2007; Rosa Neto et al., 2010). A capacidade motora é considerada um dos melhores indicadores do bem-estar global da criança, representando, dentre as áreas do desenvolvimento, a de mais fácil observação. Como os problemas de coordenação e controle do movimento poderão se prolongar até a fase adulta, crianças com desenvolvimento motor atípico, ou com risco de atrasos, merecem atenção e ações específicas (Baltieri et al., 2010; Willrich et al., 2009).

A identificação precoce dos riscos para o atraso do desenvolvimento motor permite que as crianças que nasceram de parto prematuro e que apresentam alto risco para alterações do desenvolvimento motor recebam intervenções apropriadas de forma precoce para melhorar suas atividades de vida diária e reduzir as consequências, proporcionando uma melhor qualidade de vida. Existem poucos estudos que correlacionam o GMA com a identificação das dificuldades motoras características do TDC em crianças com idade pré-escolar.

Considerando a alta prevalência das dificuldades motoras, especialmente em prematuros, e o impacto negativo sobre o desempenho na vida escolar e ao longo da vida, torna-se importante identificar alterações da movimentação espontânea dos bebês nos seus primeiros meses de vida. 


\section{OBJETIVOS}

\subsection{Objetivo Geral}

Identificar a associação entre o repertório da movimentação espontânea de bebês nascidos de parto prematuro com as dificuldades motoras observadas em idade pré-escolar.

\subsection{Objetivos Específicos}

- Verificar a validade preditiva do General Movement Assessment para o desfecho do desempenho motor por meio do MABC-2.

- Estimar a ocorrência de alteração do desempenho motor por meio do MABC-2;

- Verificar a associação das variáveis clínicas e sociodemográficas com o desempenho motor. 


\section{MATERIAIS E MÉTODO}

\subsection{Tipo de Estudo}

Trata-se de um estudo longitudinal prospectivo.

\subsection{Local do Estudo}

O estudo foi realizado no Laboratório de Investigação Neurofuncional Neonatal e Pediátrica, localizado no Departamento de Fonoaudiologia, Fisioterapia e Terapia Ocupacional (FOFITO) da Universidade de São Paulo - USP.

\subsection{População}

Crianças entre 3 e 5 anos, que nasceram de parto prematuro no Hospital Universitário da USP (HU-USP), e que foram submetidas à avaliação prévia pelo GMA nas fases pré-termo, $1^{\circ}$ e/ou $3^{\circ}$ mês de vida no Ambulatório de Estimulação do Desenvolvimento do Serviço de Fisioterapia do HU-USP.

\subsubsection{Amostra}

Para determinação da amostra necessária, foi realizado o cálculo do tamanho amostral utilizando o software G.Power 3.1. Considerou-se o tamanho de 
efeito para o tempo 0.66. O poder estatístico adotado foi 0.80 e o alfa foi 0,05 . Desta forma, o tamanho amostral estimado foi de 40 crianças.

\subsubsection{Critérios de Inclusão}

- Idade gestacional < 37 semanas;

- Ambos os sexos;

- Idade entre 3 e 5 anos;

- GMA realizada nas fases pré-termo, $1^{\circ}$ e/ou $3^{\circ}$ mês de vida;

- Concordância da criança na participação do estudo pelo Termo de Assentimento;

- Aceitação dos pais e/ou responsáveis da participação no estudo e assinatura do Termo de Consentimento Livre e Esclarecido (TCLE);

\subsubsection{Critérios de Exclusão}

- Apresentar história ou diagnóstico de prejuízos motores de origem neurológica e/ou genética tais como: paralisia cerebral, autismo, síndrome de Down e outros;

- Infecções virais maternas durante a gestação;

- Malformações congênitas;

- Apresentar relato ou exames indicando prejuízos sensoriais: visual e/ou auditivo;

- Não colaboração durante o processo de avaliação. 


\subsection{Procedimentos e Materiais}

Foram selecionadas crianças do banco de dados de um estudo prévio do Laboratório de Investigação Neurofuncional Neonatal e Pediatria (LINNP-USP), que nasceram de parto prematuro (Idade gestacional $<37$ semanas), entre agosto de 2013 e setembro de 2015. Todas as crianças selecionadas possuem em anexo no prontuário avaliação pelo GMA realizada nas fases pré-termo, primeiro e/ou terceiro mês de vida. Após a identificação dessas crianças foi realizado contato com a família através de telefone ou por entrevista durante a consulta pediátrica no Hospital Universitário.

Após o primeiro contato foi agendada uma avaliação no laboratório. Os responsáveis que concordaram em participar da pesquisa assinaram o Termo de Consentimento Livre e Esclarecido (ANEXO 1), aprovado pelo Comitê de Ética e Pesquisa do Hospital Universitário da USP CEP-HU/USP sob o número 1704/18 (ANEXO 2).

As crianças foram submetidas a uma única avaliação: no primeiro momento foi realizada uma conversa com a criança explicando de forma clara os objetivos e as atividades realizadas. Após a aceitação da criança pelo Termo de Assentimento (ANEXO 3), foram realizadas anamnese e avaliação do nível socioeconômico por renda per capita, e por fim, avaliação motora por meio do MABC-2. Todo processo de avaliação teve duração média de uma hora e meia. O avaliador responsável pela aplicação do MABC-2 não tinha conhecimento dos dados pregressos das crianças em relação ao GMA para não tendenciar os resultados do teste motor. 


\subsubsection{Avaliação pelo General Movement Assessment (GMA)}

Os dados do GMA foram retirados do banco de dados do LINNP-USP. A avaliação ocorreu durante o período de internação ou durante as primeiras consultas pediátricas pós alta hospitalar, como rotina de aplicação do GMA no Hospital Universitário, nos primeiros dias de vida do recém nascido (RN).

Os GMs foram filmados nos RNs e lactentes, de preferência uma hora antes da mamada, em estado de vigília ativa - estado 4 (Prechtl, 1974), por um período de 2 a 3 minutos. Foram avaliados na posição supina, apenas com fralda ou roupas pequenas (bodies), o que não limitou a movimentação espontânea e permitiu a visualização completa dos movimentos de membros superiores e inferiores.

Para realização das filmagens foi utilizada uma câmera filmadora portátil posicionada a uma distância que permitia a observação de todo o corpo do lactente (a 80 centímetros). Nenhuma interação do mesmo com o examinador, com pessoas próximas a ele ou com brinquedos foi permitida e em casos de agitação e choro as gravações foram interrompidas. As avaliações foram realizadas em dois momentos:

1. Fase dos Writhing movements: os GMs na fase dos WM foram avaliados nos RNs e lactentes em consultas pré-agendadas entre a segunda e a quinta semana de idade gestacional corrigida.

2. Fase dos Fidgety movements: os GMs na fase dos FM foram avaliados nos lactentes em consultas pré-agendadas no terceiro mês (entre a $12^{\mathrm{a}}$ e a $15^{\mathrm{a}}$ semana) de idade gestacional corrigida.

A análise das filmagens foi realizada por uma fisioterapeuta com certificação internacional com nível avançado pelo método qualitativo dos GMs, que classificou o tipo dos movimentos observados. Foram classificados da seguinte forma: 
Fase dos Writhing movements: normal ou alterado

Fase dos Fidgety movements: normal ou alterado

\subsubsection{Avaliação motora - Movement Assessment Battery for Children 2}

A avaliação motora foi realizada no Laboratório de Investigação Neurofuncional Neonatal e Pediátrico localizado na FOFITO USP, em uma sala clara, arejada e sem objetivos que provocassem distração para as crianças durante o procedimento de avaliação. A sala era composta por uma mesa e duas cadeiras para a aplicação dos testes de destreza manual.

Após o preenchimento do TCLE pelos pais e/ou responsáveis e o Termo de Assentimento pela criança, concordando em participar da pesquisa, foi realizado o procedimento de avaliação que durou em média uma hora e meia.

A primeira etapa da avaliação foi composta pela anamnese que consistia em uma síntese da história clínica e pessoal da criança com informações referentes à gestação (idade da mãe no período da gestação, uso de álcool, remédios ou substâncias correlatas, complicação durante e após o parto, amamentação, etc.), saúde da criança (doenças, fraturas, cirurgias sofridas, uso de medicamentos, alterações neurológicas, acompanhamento médico, etc.), internações e se a criança havia realizado acompanhamento fisioterapêutico em algum momento do desenvolvimento motor. Na história pessoal ou referente à família, buscaram-se informações quanto à cultura, raça, etnia e condições socioeconômicas (religião, idioma, cor da pele, naturalidade, nacionalidade, estado civil e escolaridade dos pais, moradia, renda, etc.) (ANEXO 4). 
O desempenho motor foi avaliado por meio do MABC-2, por uma avaliadora treinada e com experiência na aplicação do método. O MABC-2 é utilizado para identificar dificuldades motoras em crianças e adolescentes na faixa etária entre três

e 16 anos. É composto por oito tarefas que exigem três classes de habilidades: Destreza manual, Mirar e Receber, e Equilíbrio. As tarefas são divididas em três faixas: 3 a 6 anos (banda 1); 7 a 10 anos (banda 2); 11 a 16 anos (banda 3). No presente estudo utilizamos a banda 1. O teste classifica as crianças de acordo com o grau de dificuldade motora.

Os escores padrão variam de 1 a 19 e para cada valor existe o percentil correspondente, que varia de $0,1 \%$ a $99,9 \%$. Pontuações com valores iguais ou inferiores ao $5^{\circ}$ percentil referem-se ao ponto de corte para dificuldades motoras severas, podendo ser classificadas em: dificuldade no movimento; risco de dificuldade; ou não possui nenhuma dificuldade. Crianças que pontuam entre o $6^{\circ} \mathrm{e}$ o $15^{\circ}$ percentil são consideradas em risco de ter dificuldade motora, e aquelas com valores iguais ou superiores ao $16^{\circ}$ percentil são consideradas na faixa de desenvolvimento típico (ANEXO 5).

\subsection{Análise estatística}

As características clínicas e sociodemográficas dos participantes (mães e das crianças), como: realização de pré-natal, presença de complicações durante a gestação, tipo de parto, intercorrências durante o parto, idade gestacional, peso ao nascimento, pontuação do Apgar, necessidade de suporte ventilatório, foram analisadas de forma descritiva com média e desvio padrão. Foi utilizado o teste One 
Way Anova para observar se houve diferença entre o grupo de crianças com e sem dificuldades motoras. Para todos os testes realizados, foram consideradas crianças com dificuldades motoras aquelas com percentil $\leq 5 \%$ no MABC- 2 .

Foi realizado cálculo de sensibilidade, especificidade, valores preditivos positivos e negativos para a predição do GMA para o desfecho do desempenho motor pelo MABC-2, considerando uma acurácia e intervalo de confiança de 95\%. Para avaliar a validade preditiva entre as avaliações os resultados foram dicotomizados entre normais e anormais.

Para verificar a relação e interação entre a prematuridade e os resultados dos GMs (diferentes categorias na fase dos WM e FM) com a pontuação padronizada pela idade do MABC-2 (variável dependente) foi realizada regressão logística utilizando o teste Fisher exact, considerando $\mathrm{p}<0,05$ efeito estatisticamente significativo.

As análises estatísticas foram realizadas no software Rstudio versão 2018. 


\section{RESULTADOS}

\subsection{Casuística}

Das crianças identificadas no banco de dados do laboratório, 23 preenchiam todos os critérios de inclusão e participaram da pesquisa atual e foram submetidas às duas avaliações: pelo GMA durante o período de internação e/ou pós-alta hospitalar e pelo MABC-2 com idade pré-escolar. A Figura 2 representa o fluxograma do processo de seleção e inclusão dos participantes.

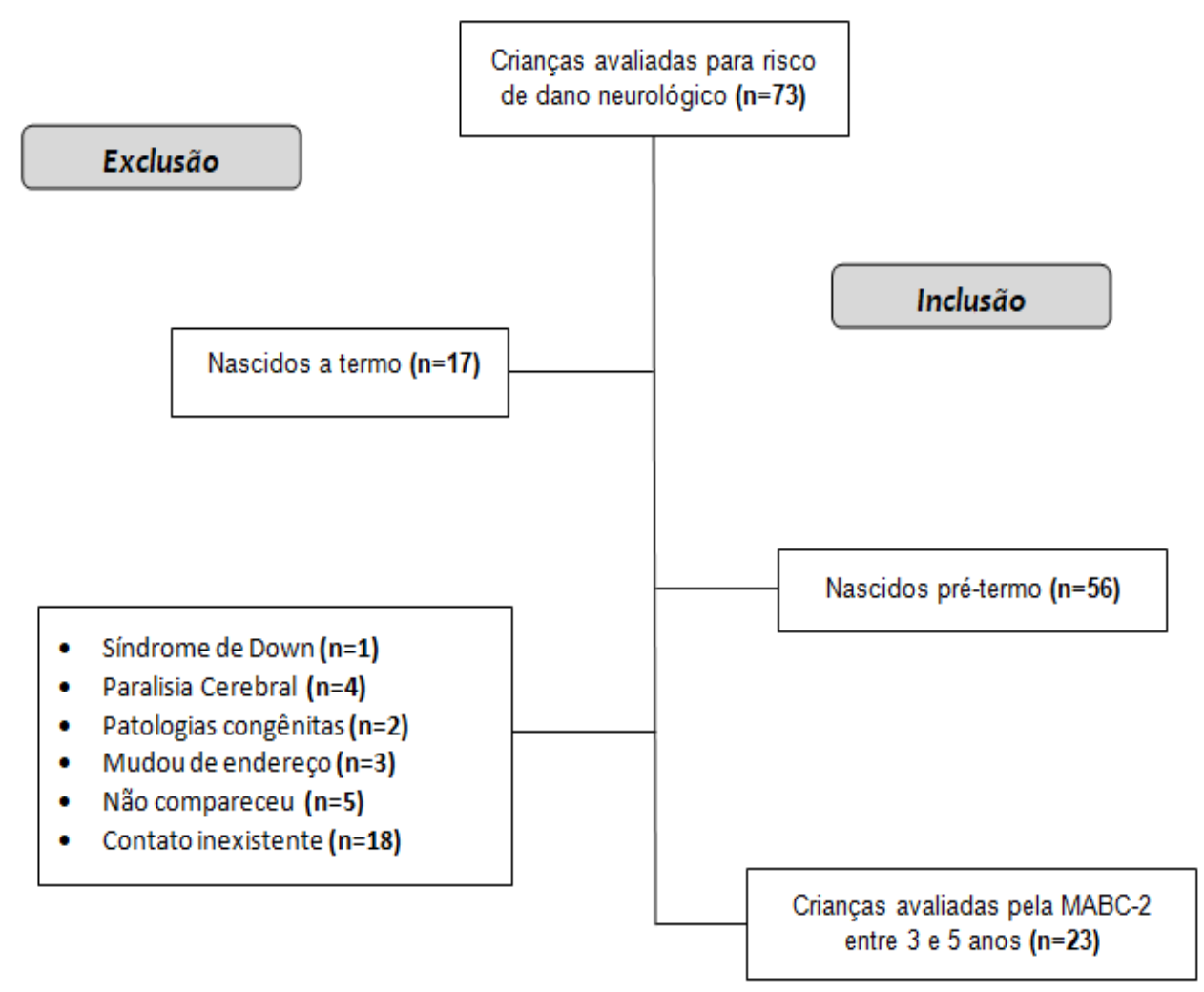

Figura 2: Fluxograma do processo de seleção e inclusão dos participantes. 
Foram avaliados o desempenho motor de 23 crianças em idade pré-escolar pelo MABC-2 (65,3\% eram do gênero masculino; 47,8 eram prematuros tardios; $39,2 \%$ apresentaram muito baixo peso ao nascer) (Tabela 1 ).

A maioria das mães com idade igual ou superior a 35 anos $(52,2 \%)$ e apresentava ensino médio completo $(n=15 ; 65,2 \%)$.

Tabela 1 - Caracterização da amostra: variáveis clínicas e sociodemográficas

\begin{tabular}{lcc}
\hline Variáveis & $\boldsymbol{n}$ & $\%$ \\
\hline Gênero (masculino) & 15 & 65,3 \\
Etnia (não-caucasiano) & 12 & 52,2 \\
IG (prematuridade tardia) & 11 & 47,8 \\
Peso ao Nascer (muito baixo peso) & 9 & 39,2 \\
Apgar 1 min (8-10) & 12 & 52,2 \\
Apgar 5 min (8-10) & 16 & 69,6 \\
Tônus muscular (normotonia) & 21 & 91,3 \\
Fisioterapia motora (sim) & 12 & 52,2 \\
GMA - Pré-termo + WM (normal) & 12 & 52,2 \\
GMA - FM (normal) & 17 & 73,9 \\
Idade materna ( $\geq 35$ anos) & 12 & 52,2 \\
Escolaridade materna (médio completo) & 15 & 65,2 \\
Escolaridade paterna (médio completo) & 13 & 56,5 \\
Renda familiar (2.000,00 - 2.999,00 R $\$$ ) & 8 & 34,7 \\
\hline Total de crianças & $\mathbf{2 3}$ & $\mathbf{1 0 0 \%}$ \\
\hline
\end{tabular}

Legenda: IG = Idade Gestacional; GMA = General Movements Assessment; $\mathrm{WM}=$ Writing Movements; FM= Fidgety Movements.

\subsection{Resultados descritivos dos General Movements e do MABC-2}

As 23 crianças que realizaram avaliação do seu desempenho motor em idade pré-escolar apresentavam resultados prévios da avalição da movimentação 
espontânea pelo GMA. Em relação aos resultados da movimentação espontânea pelo GMA na fase dos writhing movements, 12 (52,17\%) apresentaram movimentação normal. Na fase dos fidgety movements 17 (73,9\%), observamos uma tendência de normalização da movimentação espontânea nas avaliações, quando comparamos os resultados em ambas as fases (Tabela 2).

Tabela 2 - Resultados da avaliação pelo GMA

\begin{tabular}{ccccc}
\hline & Writhing Movements & \multicolumn{2}{c}{ Fidgety Movements } \\
\hline Avaliação GMA & $\mathbf{n = 2 3}$ & $\%$ & $\mathbf{n = 2 3}$ & $\%$ \\
normal & 12 & 52,2 & 17 & 73,9 \\
alterado & 11 & 47,8 & 6 & 26,1 \\
\hline
\end{tabular}

Legenda: GMA = General Movements Assessment.

Já na identificação das dificuldades motoras por meio do MABC-2 em idade pré-escolar, foram utilizados o percentil total e o percentil em cada uma das classes de habilidades da bateria (destreza manual, mirar e receber, e equilíbrio) para identificação das dificuldades motoras, considerados como valores $\leq 5 \%$ (Tabela 3).

A maior parte das crianças avaliadas apresentou resultados $\leq 5 \%$ no percentil total do MABC-2, o que corresponde a 15 crianças $(65,3 \%)$, classificadas com dificuldade motora.

Considerando apenas o domínio de destreza manual, 11 (47,8\%) crianças apresentaram dificuldade motora com escore $\leq 5 \%$. Em relação à habilidade mirar e receber, $18(78,3 \%)$ foram classificadas sem dificuldade motora, a única habilidade que a maioria das crianças apresentaram percentil $>5 \%$. Já no domínio equilíbrio, 17 
$(73,9 \%)$ crianças com percentil $\leq 5$ foram classificadas com dificuldade motora (Tabela 3).

Tabela 3 - Resultados da avaliação pelo MABC-2

\begin{tabular}{lcccc}
\hline & \multicolumn{5}{c}{ MABC-2 } & \multicolumn{3}{c}{$\mathbf{5 \%}$} \\
\hline \multirow{2}{*}{ Domínios } & $\boldsymbol{N}$ & $\mathbf{5 \%}$ & $\boldsymbol{n}$ & $\%$ \\
\cline { 2 - 5 } & 11 & 47,8 & 12 & 52,2 \\
Destreza Manual & 5 & 21,7 & 18 & 78,3 \\
Mirar e Receber & 17 & 73,9 & 6 & 26,1 \\
Equilíbrio & 15 & 65,2 & 8 & 34,8 \\
Total & & & & \\
\hline
\end{tabular}

Legenda: MABC-2 = Movement Assessment Battery for Children Second Edition

\subsection{Validade preditiva do General Movement Assessment para o desfecho motor pelo MABC-2}

Comparando os resultados do desempenho motor pelo MABC-2 com as avaliações na fase dos writhing movements, $8(34,7 \%)$ crianças que apresentaram movimentação espontânea anormal também apresentaram escores abaixo de $<5 \%$ em relação ao desempenho motor pelo MABC-2 $(p=0,640)$. Na mesma fase, 12 crianças que apresentaram movimentação espontânea normal 5 (21,7\%) evoluíram com um desfecho abaixo do esperado para a idade em relação ao desempenho motor pelo MABC-2 (Tabela 4).

Já na fase dos fidgety movements, das 17 crianças que apresentaram movimentação espontânea normal 9 (39,2\%), tiveram um bom desfecho do 
desempenho motor pelo MABC-2. E em relação às crianças ainda na mesma fase que apresentaram movimentação espontânea alterada, 2 (8,7\%), continuaram com um desfecho ruim em relação ao desempenho motor para a idade cronológica.

Tabela 4 - Desfecho motor do GMA pelo MABC-2

\begin{tabular}{rcc}
\hline \multicolumn{3}{c}{ MABC-2 } \\
\hline & $\mathbf{5} \%$ & $\mathbf{> 5 \%}$ \\
& $\mathbf{n}(\%)$ & \\
\hline Writhing movements & & \\
Normal & $5(21,7 \%)$ & $7(30,4 \%)$ \\
Alterado & $8(34,7 \%)$ & $3(13,2 \%)$ \\
Fidgety movements & & \\
Normal & $8(34,7 \%)$ & $9(39,2 \%)$ \\
alterado & $4(17,4 \%)$ & $2(8,7 \%)$ \\
\hline
\end{tabular}

Legenda: MABC-2 = Movement Assessment Battery for Children Second Edition

De acordo com a figura 3, podemos observar a relação da movimentação espontânea na fase dos writhing movements com os resultados pelo MABC-2 nas classes de habilidades: destreza manual, mirar e receber, equilíbrio e valor total. Lactentes que apresentaram movimentação espontânea na fase dos writhing movements anormal, tiveram resultados com escores $\leq 5 \%$ pelo MABC-2 na maioria das avaliações. Ainda em relação às avaliações pelo GMA na fase dos writhing movements, observamos que as crianças com movimentação normal, apresentaram resultados com escores $\geq 5 \%$ no domínio mirar e receber. 


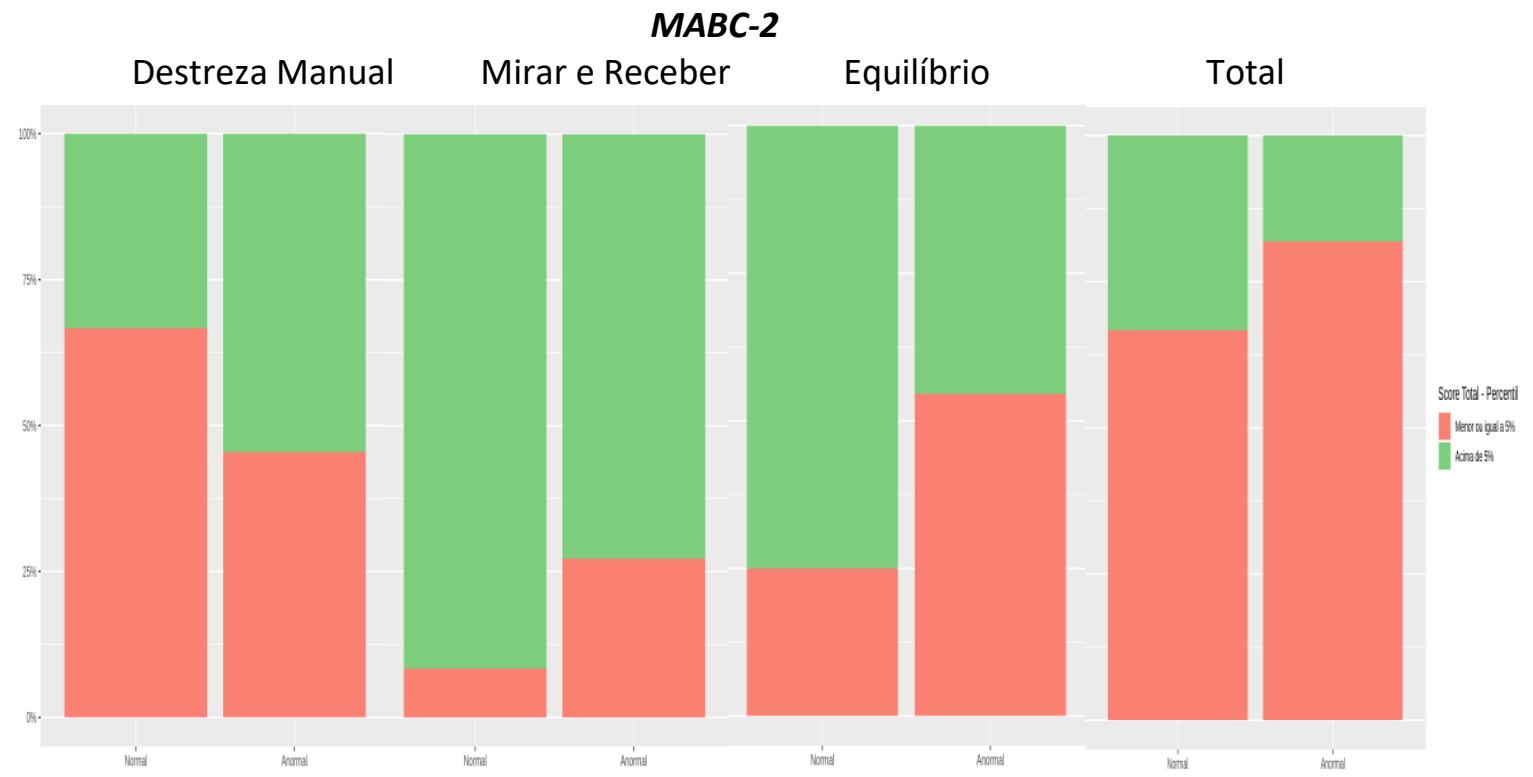

Figura 3: Comparação da classificação na fase dos writhing movements pelo GMA com o Percentil total pela MABC-2.

Na figura 4, observamos os resultados pelo GMA com os domínios do MABC2, nas classes de habilidade: destreza manual, mirar e receber, equilíbrio e total, em relação aos resultados classificados como normal e anormal na fase dos fidgety movements.

\section{MABC-2}

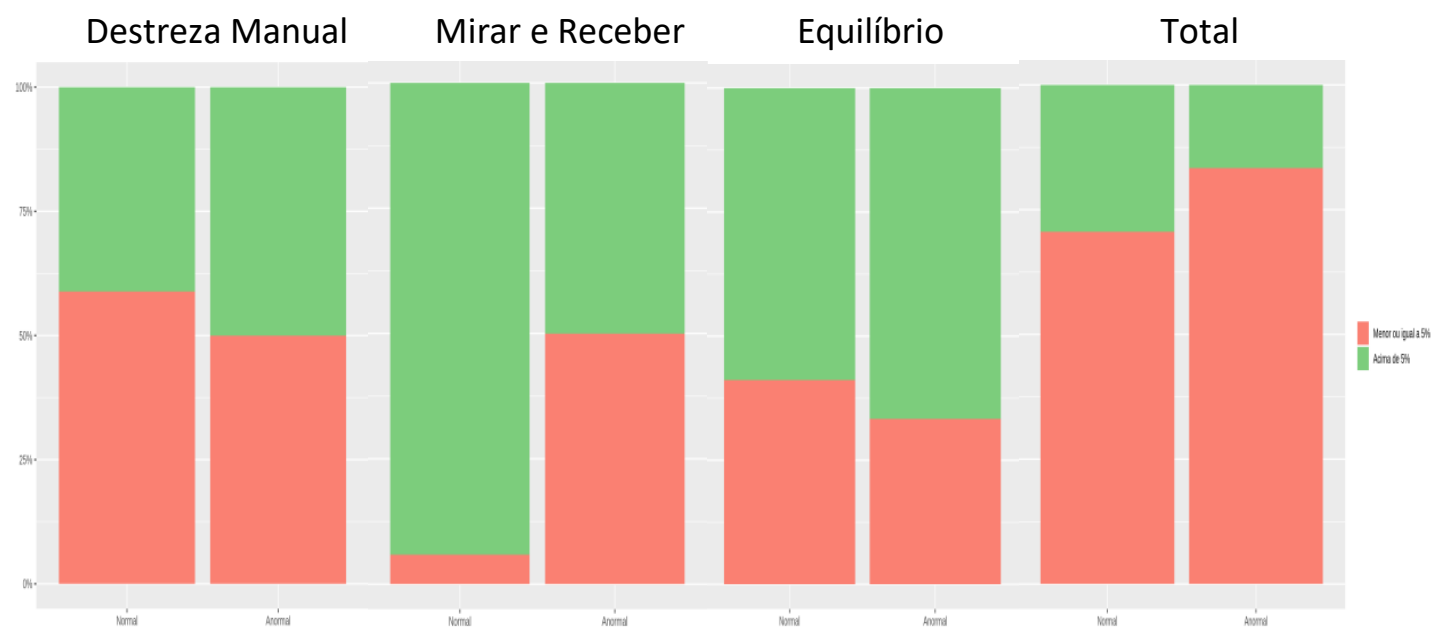

Figura 4: Comparação de todos os domínios do MABC-2 em relação aos resultados obtidos na fase dos fidgety movements pelo GMA. 
Valores de sensibilidade, especificidade, valores preditivos positivos e negativos entre as duas avaliações (GMA e MABC-2) são apresentados na tabela 5.

Podemos observar que os GMs não foram preditivos para o desfecho motor pelo MABC-2. Foi encontrado um valor preditivo negativo de 0,90 em relação à fase dos fidgety movements com o MABC-2, indicando que são confiáveis para prever desenvolvimento normal.

Tabela 5 - Valores preditivos do GMA para o desfecho motor pelo MABC-2.

\begin{tabular}{lcc}
\hline & WM x MABC-2 & FM x MABC-2 \\
\hline Sensibilidade & $27 \%$ & $53 \%$ \\
Especificidade & $75 \%$ & $62 \%$ \\
Valor preditivo positivo & 0,67 & 0,73 \\
Valor preditivo negativo & 0,35 & 0,90 \\
\hline
\end{tabular}

Legenda: WM - writhing movements; FM - fidgety movements.

\subsection{Influência dos fatores clínicos e sociodemográficos no desempenho motor}

A caracterização entre grupos em relação às variáveis clínicas é apresentada na Tabela 6. Para as variáveis binárias foi realizada regressão logística com corte em 5\% a partir do Percentil pelo MABC-2, para considerar os resultados obtidos como normais ou dificuldade motora nessa população. 
No presente estudo mais meninos 10 (66,6\%; Tabela 6), do que meninas apresentaram escores abaixo $\leq 5 \%$ na avaliação do desempenho motor pelo Percentil Total do MABC-2, entretanto, o sexo masculino não afeta de forma significativa o resultado do desempenho motor (OR:3,33; p:0,369; 95\% IC:0.27188.27) (Tabela 6).

Os resultados mostraram que quanto menor a idade gestacional, peso ao nascer, Apgar de $1^{\circ}$ e $5^{\circ}$ minuto maior será o risco das crianças apresentar escores baixos nas classes de habilidades: destreza manual, equilíbrio e escore total pelo MABC-2.

Crianças que realizaram fisioterapia motora durante alguma fase do desenvolvimento motor apresentaram escores abaixo $\leq 5 \%$ pelo MABC-2, porém esses resultados não são estatisticamente significativos.

Tabela 6 - Caracterização entre grupos em relação às variáveis clínicas.

\begin{tabular}{|c|c|c|c|}
\hline Variável & $\leq 5 \%$ & $>5 \%$ & $p$ \\
\hline Sexo (masculino) & $10(66,6 \%)$ & $5(33,3 \%)$ & 0,369 \\
\hline IG ( $(\leq 230$ dias $)$ & $7(46,6 \%)$ & $1(6,7 \%)$ & 0,341 \\
\hline Peso ( $\leq 1.599$ gramas) & $8(53,4 \%)$ & 0 & 0,341 \\
\hline Apgar $1^{\circ}$ minuto $(1-6)$ & $6(40 \%)$ & 0 & 0,369 \\
\hline Apgar $5^{\circ}$ minuto (1-6) & $3(20 \%)$ & 0 & 0,539 \\
\hline Realizou Fisioterapia & $6(40 \%)$ & $2(13,3 \%)$ & 1 \\
\hline WM Anormal & $6(40 \%)$ & $1(6,6 \%)$ & 0.640 \\
\hline FM Anormal & $3(20 \%)$ & $1(6,6 \%)$ & 1 \\
\hline
\end{tabular}

Legenda: $I G=$ Idade Gestacional; GMA = General Movements Assessment; WM = Writing Movements; FM= Fidgety Movements. 
Já a correlação das variáveis tônus e fidgety movements aparecem como protetivas para dificuldades motoras pelo MABC-2. Crianças que apresentaram pontuações acima de $\geq 5 \%$ pelo $M A B C-2$, tiveram movimentação normal na avaliação pelo General Movement Assessment na fase dos fidgety movements ( $O R=0,07 ; p: 0,040 ; 95 \% \mathrm{IC}: 0.001-1.256)$. As crianças avaliadas com tônus normal, tiveram melhores resultados no MABC-2 (OR:0; p:0,023; 95\%IC:0.000-0.968).

Dentre as variáveis socioeconômicas, a que influenciou diretamente nos resultados obtidos pela MABC-2 foi à escolaridade materna. Nos resultados de destreza manual, observamos que crianças com melhores resultados neste domínio, são filhos de mães com maior grau de escolaridade $(p=0.035)$ (Tabela 7). 
Tabela 7 - Resultados do MABC-2 em relação as variáveis clínicas e sociodemográficas.

\begin{tabular}{|c|c|c|c|c|c|c|c|c|c|c|c|c|}
\hline & \multicolumn{3}{|c|}{ Destreza Manual } & \multicolumn{3}{|c|}{ Mirar e Receber } & \multicolumn{3}{|c|}{ Equilíbrio } & \multicolumn{3}{|c|}{ Total } \\
\hline & $<5 \%$ & $\geq 5 \%$ & & $<5 \%$ & $\geq 5 \%$ & & $<5 \%$ & $\geq 5 \%$ & & $<5 \%$ & $\geq 5 \%$ & \\
\hline & M (DP) & $\mathrm{M}$ (DP) & $\mathbf{P}$ & M (DP) & $M(D P)$ & $\mathbf{P}$ & $M(D P)$ & $\mathrm{M}(\mathrm{DP})$ & $\mathbf{P}$ & $M$ (DP) & $M(D P)$ & $\mathbf{P}$ \\
\hline \multicolumn{13}{|l|}{ Gênero } \\
\hline Feminino & $3 \pm 1$ & $6.7 \pm 2.8$ & & $4 \pm 0$ & $8.7 \pm 3.1$ & & $4 \pm 0$ & $5.9 \pm 2.6$ & & $3.6 \pm 0.4$ & $5.4 \pm 2.4$ & \\
\hline Masculino & $2.5 \pm 0.5$ & $7.5 \pm 2.3$ & 0.074 & $2.5 \pm 0.5$ & $9.2 \pm 2.3$ & 1 & $3.3 \pm 0.7$ & $7.5 \pm 2.6$ & 0.399 & $3 \pm 0.8$ & $7.2 \pm 2.1$ & 0.369 \\
\hline \multicolumn{13}{|l|}{ Idade Gestacional } \\
\hline$\leq 230$ dias & $2.6 \pm 0.7$ & $6.7 \pm 1.0$ & & $3.5 \pm 0.5$ & $8.9 \pm 1.6$ & & $3.2 \pm 0.7$ & $7.1 \pm 2.0$ & & $3 \pm 0.8$ & $6.4 \pm 0.8$ & \\
\hline$\geq 231$ dias & - & $6.7 \pm 2.9$ & 0.685 & $2 \pm 0$ & $10.5 \pm 2.4$ & 1 & - & $7 \pm 2.4$ & 0.196 & $3.6 \pm 0.4$ & $7.5 \pm 1.9$ & 0.341 \\
\hline \multicolumn{13}{|l|}{ Peso ao Nascer } \\
\hline$\leq 1.599$ gramas & $2.6 \pm 0.7$ & $6.8 \pm 0.9$ & 0.221 & $3 \pm 0.8$ & $9 \pm 1.7$ & 0.603 & $3.4 \pm 0.7$ & $6.5 \pm 1.6$ & 0.196 & $3.1 \pm 0.8$ & $6.2 \pm 0.8$ & 0.341 \\
\hline$\geq 1.600$ gramas & - & $6.6 \pm 2.9$ & & - & $8.6 \pm 3.1$ & & - & $6.2 \pm 2.6$ & & $3.5 \pm 0.5$ & $7.5 \pm 1.8$ & \\
\hline \multicolumn{13}{|l|}{ Raça } \\
\hline Caucasiano & $3 \pm 0.8$ & $8 \pm 2.8$ & 1 & $2.5 \pm 0.5$ & $9.3 \pm 2.2$ & 0.316 & $3.3 \pm 0.4$ & $6.7 \pm 2.4$ & 1 & $3.3 \pm 0.7$ & $6.6 \pm 1.8$ & 0.370 \\
\hline Não-caucasiano & $2.3 \pm 0.4$ & $7.4 \pm 1.1$ & & $4 \pm 0$ & $9.9 \pm 2.1$ & & $3.5 \pm 0.8$ & $7.3 \pm 2.1$ & & $3 \pm 0.8$ & $7.4 \pm 1.4$ & \\
\hline \multicolumn{13}{|l|}{ Apgar $1^{\circ}$ minuto } \\
\hline $7-10$ & $4 \pm 0$ & $8.0 \pm 2.1$ & 0.687 & $2 \pm 0$ & $10.2 \pm 2.1$ & 0.102 & $3 \pm 0.8$ & $7.2 \pm 2.3$ & 0.657 & $4 \pm 0$ & $7.3 \pm 1.7$ & 0.369 \\
\hline $0-6$ & $2.4 \pm 0.4$ & $6 \pm 1.4$ & & $3.5 \pm 0.5$ & $8.1 \pm 1.5$ & & $3.7 \pm 0.4$ & $6.5 \pm 2.0$ & & $2.5 \pm 0.5$ & $6 \pm 1$ & \\
\hline \multicolumn{13}{|l|}{ Apgar $5^{\circ}$ minuto } \\
\hline $7-10$ & $2.3 \pm 0.4$ & $7.8 \pm 2.1$ & 1 & $2.5 \pm 0.5$ & $10 \pm 2.1$ & 1 & $3 \pm 0.7$ & $7.2 \pm 2.3$ & 0.260 & $3.2 \pm 0.8$ & $7.0 \pm 1.7$ & 0.539 \\
\hline $0-6$ & $3 \pm 0.8$ & $5 \pm 0$ & & - & $8.2 \pm 2.0$ & & $4 \pm 0$ & $5 \pm 0$ & & $3 \pm 0.7$ & - & \\
\hline \multicolumn{13}{|l|}{ Tônus } \\
\hline Normal & $2.6 \pm 0.7$ & $8.0 \pm 2.0$ & 0.177 & $3 \pm 1$ & $10.1 \pm 1.8$ & $0.021^{*}$ & $3.5 \pm 0.7$ & $7.2 \pm 2.3$ & 1 & $3.3 \pm 0.8$ & $7.0 \pm 1.7$ & 1 \\
\hline Alterado & - & $5 \pm 0$ & & $3 \pm 0$ & $5 \pm 0$ & & $3 \pm 0$ & $5 \pm 0$ & & $5.3 \pm 2.5$ & - & \\
\hline \multicolumn{13}{|c|}{$\begin{array}{l}\text { General Movement - } \\
\text { termo + WM }\end{array}$} \\
\hline Normal & $3 \pm 0$ & $7.1 \pm 1.3$ & 0.413 & - & $8.7 \pm 3.0$ & 0.316 & $3 \pm 1$ & $6.6 \pm 1.8$ & 0.213 & $3.5 \pm 0.5$ & $7 \pm 1.6$ & 0.640 \\
\hline Alterado & $2.5 \pm 0.8$ & $7.8 \pm 1.2$ & & $3 \pm 0.8$ & $9.6 \pm 1.8$ & & $3.6 \pm 0.4$ & $7.8 \pm 2.7$ & & $3 \pm 0.9$ & $7.8 \pm 1.9$ & \\
\hline
\end{tabular}




\begin{tabular}{|c|c|c|c|c|c|c|c|c|c|c|c|c|}
\hline \multicolumn{13}{|l|}{ General Movement - FM } \\
\hline Normal & $3 \pm 0.7$ & $7.5 \pm 1.8$ & 1 & - & $8.7 \pm 3.0$ & $0.040^{*}$ & $3.4 \pm 0.8$ & $6.5 \pm 1.6$ & 1 & $3.4 \pm 0.7$ & $6.8 \pm 1.6$ & 1 \\
\hline Alterado & $2 \pm 0$ & $8.2 \pm 2.9$ & & $3 \pm 0.8$ & $9 \pm 0.8$ & & $3.5 \pm 0.5$ & $8.5 \pm 3.2$ & & $2.7 \pm 0.8$ & $8.5 \pm 1.5$ & \\
\hline \multicolumn{13}{|l|}{ Fisioterapia } \\
\hline Sim & $2.6 \pm 0.7$ & $8.1 \pm 3.1$ & 0.213 & \pm & $8.7 \pm 3.0$ & 0.316 & $3.8 \pm 0.4$ & $6.7 \pm 2.6$ & 1 & $3 \pm 0.7$ & $7 \pm 1.6$ & 1 \\
\hline Não & \pm & $6.7 \pm 2.8$ & & $3 \pm 0.8$ & $10.2 \pm 2.5$ & & $2.5 \pm 0.5$ & $7.3 \pm 2$ & & $3.5 \pm 0.8$ & $7.1 \pm 1.7$ & \\
\hline \multicolumn{13}{|l|}{ Idade Materna } \\
\hline$\leq 34$ anos & $2.6 \pm 0.4$ & $7.3 \pm 2.1$ & 0.654 & $3 \pm 0$ & $9.3 \pm 2.0$ & 0.938 & $3.2 \pm 0.7$ & $6.8 \pm 1.4$ & 0.183 & $3 \pm 0.8$ & $6 \pm 0.8$ & 0.282 \\
\hline$\geq 35$ anos & $2 \pm 0$ & $8 \pm 2.1$ & & $3 \pm 1$ & $10 \pm 2.3$ & & $4 \pm 0$ & $7.2 \pm 2.6$ & & $3.3 \pm 0.7$ & $8.1 \pm 1.6$ & \\
\hline \multicolumn{13}{|l|}{ Escolaridade Materna } \\
\hline Ensino Fund. Incomp & - & - & 0.035 & - & - & 0.268 & - & - & 0.926 & & & 0.348 \\
\hline Ensino Fund. Comp. & - & $6.7 \pm 2.8$ & & $2 \pm 0$ & $11.6 \pm 1.8$ & & - & $6.2 \pm 2.6$ & & $4 \pm 0$ & $7 \pm 0$ & \\
\hline Ensino Médio Incomp. & \pm & $7 \pm 0$ & & - & $9 \pm 0$ & & - & $6 \pm 0$ & & - & $6 \pm 0$ & \\
\hline Ensino Médio Comp. & $3 \pm 0.8$ & $8.3 \pm 2.5$ & & $3.5 \pm 0.5$ & $9.9 \pm 1.7$ & & $3.6 \pm 0.4$ & $8.5 \pm 2.4$ & & $2.8 \pm 0.8$ & $7.2 \pm 1.9$ & \\
\hline Ensino Superior Incomp. & \pm & $5 \pm 0$ & & - & $5 \pm 0$ & & - & $5 \pm 0$ & & $3 \pm 0$ & - & \\
\hline Ensino Superior Comp. & $2 \pm 0$ & $8 \pm 0$ & & - & $7.5 \pm 1.5$ & & $2 \pm 0$ & $5 \pm 0$ & & $3.5 \pm 0.5$ & - & \\
\hline \multicolumn{13}{|l|}{ Escolaridade Paterna } \\
\hline Ensino Fund. Incomp. & $2.3 \pm 0.4$ & $7 \pm 0$ & 0.946 & \pm & $8.6 \pm 3.1$ & 0.519 & $4 \pm 0$ & $6 \pm 0$ & 0.451 & $2.3 \pm 0.4$ & $7 \pm 0$ & 0.172 \\
\hline Ensino Fund. Comp. & & $6.7 \pm 2.9$ & & $3 \pm 0$ & $10 \pm 2.9$ & & $3 \pm 0$ & $5.7 \pm 0.8$ & & $3 \pm 0.8$ & $7 \pm 0$ & \\
\hline Ensino Médio Incomp. & & $13 \pm 0$ & & & $10 \pm 0$ & & & $13 \pm 0$ & & & $10 \pm 0$ & \\
\hline $\begin{array}{l}\text { Ensino Médio Comp. } \\
\text { Ensino Superior Incomp. }\end{array}$ & $3.5 \pm 0.5$ & $7.9 \pm 1.7$ & & $3 \pm 1$ & $10.2 \pm 1.7$ & & $3 \pm 0.8$ & $7.2 \pm 1.8$ & & $4 \pm 0$ & $6.7 \pm 1.6$ & \\
\hline Ensino Superior Comp. & $2 \pm 0$ & \pm & & \pm & $9 \pm 0$ & & & $5 \pm 0$ & & $3 \pm 0$ & \pm & \\
\hline \multicolumn{13}{|l|}{ Renda Familiar } \\
\hline Até $1.499,00$ & $2.3 \pm 0.4$ & $7 \pm 0$ & 0.938 & \pm & $8.5 \pm 3.2$ & 0.378 & $4 \pm 0$ & $6 \pm 1$ & 0.109 & $2.7 \pm 0.8$ & $7 \pm 0$ & 0.242 \\
\hline De $1.500,00$ a $1.999,00$ & \pm & $7.1 \pm 2.9$ & & $2.5 \pm 0.5$ & $11 \pm 2.1$ & & $3 \pm 0$ & $6.6 \pm 3.0$ & & $5.6 \pm 2.4$ & $7 \pm 2.1$ & \\
\hline De $2.000,00$ a $2.999,00$ & $3.5 \pm 0.5$ & $6.6 \pm 1.1$ & & $4.0 \pm 0$ & $8.8 \pm 2.5$ & & $3 \pm 0.8$ & $6.6 \pm 1.7$ & & $3.7 \pm 0.4$ & $6.2 \pm 0.8$ & \\
\hline Acima de $3.000,00$ & $2 \pm 0$ & $7.1 \pm 2.9$ & & \pm & $8.5 \pm 3.0$ & & \pm & $5.9 \pm 2.6$ & & $3 \pm 0$ & $5.6 \pm 2.4$ & \\
\hline
\end{tabular}

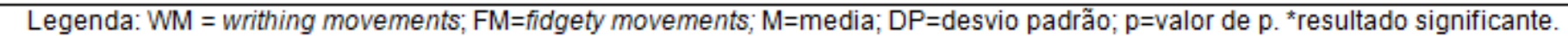




\section{DISCUSSÃO}

O objetivo do presente estudo foi identificar a associação entre repertório da movimentação espontânea de bebês nascidos de parto prematuro no HU-USP, com as dificuldades motoras observadas em idade pré-escolar. A maioria dos bebês avaliados apresentaram movimentação espontânea anormal nas fases dos writhing movements e fidgety movements pelo General Movement Assessment, e mais da metade também foram classificados abaixo do $5^{\circ}$ percentil pelo MABC-2 em idade pré-escolar, indicando baixo desempenho motor. Entretanto, a movimentação espontânea normal na fase dos fidgety movements, demonstrou ser fator protetivo para o desempenho motor na idade pré-escolar.

Estudos anteriores mostram alta validade preditiva negativa da avaliação na fase do FM para o desfecho motor. Segundo Janneke et al., (2008) bebês com FM ausente, alterados ou com repertório pobre são preditivos para desfecho motor anormal relacionado à dificuldade de coordenação motora, equilíbrio e motricidade fina; já no estudo de Spittle et al., (2013) é apontado um alto valor preditivo negativo para FM normal com desfecho motor normal.

Não encontramos no presente estudo valores importantes em relação à sensibilidade e especificidade do GMA para o desfecho motor pela MABC-2, achado importante de estudos anteriores como de Spittle et al (2013). Neste, foram avaliados pelo GMA lactentes que nasceram de parto prematuro com idade gestacional inferior a 30 semanas, e foram avaliados novamente aos 2 anos pela Escala de Bayley - III (BSID III) e aos 4 anos pelo MABC-2. Porém não foram excluídos os quadros de Paralisia Cerebral da amostra, o que pode ter tendenciado 
altos valores de sensibilidade e especificidade que já são claros na literatura na predição de paralisia cerebral pelo GMA.

Diversos estudos utilizam o MABC-2 na identificação de dificuldades motoras em crianças que nasceram de parto prematuro, porém poucos foram realizados durante a fase pré-escolar (entre 3 e 6 anos de idade). Em nossa amostra identificamos que a maioria das crianças avaliadas foram classificadas abaixo do $5 \%$ pelo MABC-2, resultado que corrobora com dados já descritos na literatura. Henderson et al., (2007) mostraram que o MABC-2 é um instrumento confiável para a identificação de dificuldades motoras em crianças entre 3 e 4 anos. $O$ estudo de Smits-Engelsman et al. (2011) aponta que a avaliação aos 3 anos de idade pelo MABC-2 tem boa sensibilidade para o desfecho motor aos 4,5 anos. Zwicker et al., (2017) encontrou valores altos de sensibilidade (79\%) e especificidade (93\%) na avaliação aos 4 anos com desfecho motor aos 8 anos pelo MABC-2. E dados do estudo mais recente do mesmo grupo apontam que avaliação pelo MABC-2 aos 3 anos apresenta sensibilidade de $89.7 \%$ e valor preditivo positivo de $38.2 \%$, indicando que há muitos falsos positivos na população estudada de alto risco para a prematuridade. Portanto, crianças com 3 anos, cuja pontuação pela MABC-2 é abaixo do $5^{\circ}$ percentil, podem ter indicativos para o TDC, e não devem ser diagnosticadas com até pelo menos 4 ou 5 anos de idade. Os pesquisadores sugerem que essas crianças devem ser acompanhadas longitudinalmente e devem participar de algum programa de intervenção motora rotineiramente até pelo menos a idade escolar.

A identificação precoce das dificuldades motoras antes da idade escolar é importante para que os profissionais da saúde e educação possam traçar planos adequados para minimizar as sequelas motoras futuras nessas crianças, porém 
existem poucos estudos sobre a validade do MABC-2 em idade pré-escolar de 3 a 5 anos, como é o caso da nossa amostra. O estudo de Griffiths et al. (2017) sugere que crianças de 4 anos com pontuação abaixo do $5^{\circ}$ percentil pela MABC-2 provavelmente apresentem comprometimento motor aos 8 anos de idade. Essas informações podem ser usadas para priorizar e melhorar os serviços de intervenção e acompanhamento de crianças prematuras.

Em nosso estudo, todas as crianças que foram identificadas com escores abaixo do $5^{\circ}$ percentil foram encaminhadas para acompanhamento fisioterapêutico de rotina de acordo com a disponibilidade e interesse da família. Segundo Edward et al. (2011), recomenda-se acompanhamento do desenvolvimento motor de crianças nascidas prematuras, pois o TDC pode surgir mais tarde na infância nesta população.

Crianças que apresentam dificuldades motoras como o TDC, com ou sem comorbidades principalmente na fase escolar, apresentam qualidade de vida significativamente mais baixa que seus pares de desenvolvimento típico. Estudos apontam que os pais relatam diferença psicossocial e nos domínios físicos em relação às crianças com desenvolvimento típico. Estes achados também aparecem nos relatórios fornecidos pelos professores, sugerindo que essas crianças apresentam mais problemas emocionais e comportamentais comparados aos seus colegas (Wuang et al., 2012; Van Den Heuvel et al., 2016), desencadeando conflitos entre os pares e sofrimento psicológico. Desta forma, a identificação precoce desses achados é importante não só em relação ao desempenho motor dessas crianças, mas também em relação aos aspectos psicossociais em que ela vive.

Está descrito na literatura que diversos fatores clínicos e socioeconômicos podem influenciar no desenvolvimento motor de uma criança, alguns desses 
achados foram encontrados no presente estudo. Observamos menores escores na avaliação pelo MABC-2 nos meninos em relação às meninas, indicando um menor desempenho motor. Esses achados corroboram com um estudo de Linsell et al. (2016), onde o gênero masculino associado ao baixo peso ao nascer foram associados a menores pontuações no MABC-2 aos 5 anos de idade em crianças que nasceram de parto prematuro, o que consiste com a hipótese sugerida por Zwicker et al. (2013), em que o gênero masculino apresenta desvantagem motora com maiores índices para o TDC. Outros estudos apontam que o gênero masculino é preditor independente para pior desfecho no desenvolvimento motor de prematuros em diferentes idades gestacionais (Romeo et al., 2016).

Observamos também que os resultados do MABC-2 variam conforme a idade gestacional; sendo que quanto menor a idade gestacional, menor o desempenho motor. Estes dados corroboram com diversos estudos sobre o desenvolvimento motor de crianças prematuras. Zhu et al. (2016) demonstraram que cada semana a menos de idade gestacional eleva em $19 \%$ os riscos das crianças apresentarem dificuldades motoras. Na mais recente revisão, Edwards et al. (2018), identificaram que o risco de dificuldades motoras em crianças nascidas de parto prematuro e prematuro extremo é 8 vezes maior em relação às crianças que nasceram a termo.

A prematuridade confere maior vulnerabilidade ao cérebro, sendo um período crítico para o desenvolvimento do seu sistema nervoso, tornando-o mais suscetível aos problemas do desenvolvimento (Als et al., 2004). A escala de Apgar aplicada logo após o nascimento mostra a vitalidade do recém-nascido, notas abaixo de 5 indicam sofrimento (asfixia) perinatal. No nosso estudo observamos uma importante relação entre o desempenho motor e notas baixas pela escala de Apgar. Em especial recém-nascidos pré-termo $<30$ semanas, peso ao nascer $<1.500$ gramas e 
que apresentam asfixia severa ao nascer (Apgar $\leq 4)$ são considerados grupo de alto risco para alterações do desenvolvimento (Diaz, Schwarcz, 1987). Padrão anormal dos GMs está relacionado com a prematuridade, baixo peso ao nascer e baixos valores de Apgar (Becaria et al., 2012).

Nos lactentes prematuros extremos, padrão anormal dos GMs também está relacionado com atraso do desenvolvimento da coordenação motora fina aos dois anos de idade (Becaria et al., 2012) além de déficits cognitivos na idade escolar (Einspieler et al., 2016). Observamos nas crianças avaliadas uma relação entre fidgety movements anormais com baixos escores pelo MABC-2 na classe de habilidade de destreza manual.

Sabemos que há evidências sobre a influência de fatores socioeconômicos (renda familiar, escolaridade dos pais e estado ocupacional), no desenvolvimento motor e cognitivo desde a primeira infância até a idade escolar (Bradley et al., 2002). Diversos estudos epidemiológicos haviam identificado influência da escolaridade materna no desenvolvimento infantil, e é possível que uma maior escolaridade dos pais promova um ambiente mais enriquecido para o desenvolvimento, minimizando as dificuldades motoras. Prematuros nascidos de mães com menor nível de escolaridade apresentaram maior risco de atraso motor durante o primeiro ano de idade na Escala Motora Infantil de Alberta (EMIA) (Pampel, 2010). As condições socioeconômicas podem influenciar a qualidade e a quantidade de estímulos ambientais oferecidos à criança. Maior renda familiar e escolaridade materna refletem sobre a melhor organização do ambiente físico e temporal, maior oportunidade de variação na estimulação diária, disponibilidade de materiais adequados e jogos para a criança, maior envolvimento verbal e emocional da mãe e melhor aproveitamento da renda no cuidado à criança, proporcionando um ambiente 
mais enriquecido para o desenvolvimento infantil (Samenoff, 1986; Andrade, et al., 2005; Pampel, 2010).

O desenvolvimento motor de bebês prematuros é diferente em relação às crianças que nascem a termo, devido à maturação do sistema nervoso central ser interrompida na fase intrauterina, gerando uma insuficiência no crescimento pós natal, diminuição de força muscular, controle postural inadequado levando ao atraso da movimentação espontânea antigravitária. Os padrões e a qualidade dos movimentos nessa população podem ser prejudicados (Samsom et al., 2001). Desta forma, as crianças que nascem de parto prematuro devem ser encaminhadas para acompanhamento e intervenção fisioterapêutica para minimizar as consequências causadas pelo parto prematuro.

Observamos na nossa amostra que as crianças que realizaram fisioterapia continuaram com pontuação no MABC-2 abaixo do $5^{\circ}$ percentil, sendo esta pontuação mais baixa do que a das crianças que não realizaram fisioterapia. Muitos trabalhos abordam o tema da importância da estimulação essencial para o desenvolvimento motor de bebês prematuros para diminuir os riscos de atraso motor e minimizar sequelas futuras. Possivelmente essas crianças que realizaram fisioterapia e continuaram com desempenho motor abaixo do esperado para idade apresentariam maiores dificuldades ou sequelas motoras do que foi observado durante a atual avaliação. De fato, na nossa amostra não detectamos casos de Paralisia Cerebral, que é esperado de acordo com a literatura, em 10 a 15\% das crianças que nascem de parto prematuro. Portanto, podemos supor que, apesar das crianças que realizaram fisioterapia possuírem pior desempenho motor que as que não realizaram, resultou em uma manifestação de disfunção motora mais amena 
que a Paralisia Cerebral, provavelmente como efeito da intervenção fisioterapêutica oportuna.

$\mathrm{Na}$ amostra não houve desistência ou avaliação com necessidade de interrupção por motivo de falta de atenção, problemas comportamentais ou habilidades motoras imaturas, como mostram alguns estudos relacionados com aplicação do MABC-2. Problemas de atenção e comportamento estão presentes em crianças de 3 a 4 anos de desenvolvimento típico, que podem se cansar facilmente e podem não conseguir se sentar atentamente (Payne e Isaacs, 1998). SmitsEngelsman et al. (2011) constataram que aproximadamente $90 \%$ das crianças avaliadas aos 3 anos estavam dispostas e capazes de executar mais da metade das tarefas do MABC-2. Contudo, todos os estudos foram realizados com crianças nascidas a termo e com desenvolvimento típico. Crianças prematuras menores de 4 anos demonstraram ter cognição mais baixa, problemas comportamentais (Clark et al., 2008), problemas emocionais (Woodward et al., 2009) e baixa função executiva (Edgin et al., 2008) em comparação com seus pares. Crianças prematuras também têm um risco para hiperatividade e distúrbios do déficit de atenção (Delobel-Ayoub et al., 2006).

Como o MABC-2 é um teste de avaliação que requer atenção e cooperação para cada item do teste, crianças prematuras extremas têm maior risco de não conseguir executar adequadamente as tarefas de cada domínio quando são comparadas com seus pares, e terão maior dificuldade de concluir a avaliação. Nosso estudo foi consistente em relação às possíveis intercorrências, o ambiente para aplicação da escala foi preparado para evitar possíveis distrações, silencioso e sem objetos que poderiam tirar a atenção. 
Assim, os resultados do estudo sugerem que crianças que nascem de parto prematuro, necessitam de investigação e acompanhamento longitudinal até a idade escolar. A identificação precoce e o acompanhamento permitem redução das complicações futuras relacionadas à prematuridade, melhora do desempenho motor, da qualidade de vida e da interação social com seus pares. 


\section{LIMITAÇÕES}

O estudo apresenta algumas limitações que devem ser comentadas: um grande número de perdas das avaliações dos primeiros meses de idade para a idade pré-escolar. Algumas crianças não continuaram o acompanhamento no ambulatório de prematuros do HU-USP, o que gerou desatualização das informações em relação a telefone, endereço e e-mail. Ainda, algumas famílias não concordaram em participar das avaliações motoras com idade pré-escolar. 


\section{CONCLUSÕES}

O presente estudo permitiu identificar que o desempenho motor normal durante a movimentação espontânea até o quinto mês de idade corrigida está relacionado ao melhor desempenho motor em idade pré-escolar de crianças que nasceram de parto prematuro. Lactentes com a movimentação espontânea normal na fase dos fidgety movements apresentaram melhor desempenho motor na avaliação pela MABC-2, evidenciando o alto valor preditivo negativo encontrado.

Identificamos dificuldade motora significativa com escores abaixo de 5\% na maioria dos prematuros avaliados em idade escolar por meio da MABC-2, o que pode estar associado nesta população à menor idade gestacional, menor índice de Apgar ao nascimento, tônus normal e ao sexo masculino. $O$ maior grau de escolaridade materna parece ser um fator protetivo para o melhor desempenho motor.

Não identificamos sequelas motoras mais graves como é o caso da Paralisia Cerebral nas crianças avaliadas, apesar do desempenho motor da maioria das crianças estarem abaixo do esperado para a idade cronológica. Fica clara a importância do acompanhamento fisioterapêutico pelo menos até a idade préescolar nas crianças que nascem de parto prematuro e que apresentam alteração na movimentação espontânea pelo General Movement Assessment. 


\section{REFERÊNCIAS BIBLIOGRÁFICAS}

Allen MC. Neurodevelopmental outcomes of preterm infantis. CurrOpinNeurol. 2008;21:123-128.

Als H, Duffy FH, McAnulty GB, Rivkin MJ, Vajapeyam S, Mulkern RV, Warfield SK, Huppi PS, Butler SC, Conneman N, Fischer C, Eichenwald EC. Early experience alters brain function and structure. Pediatrics. 2004; 113: 846-57.

American Psychiatric Association. Diagnostic and statistical manual of mental disorders - DSM V (5th ed.). Porto Alegre: Artmed, 2013.

Andrade SA, Santos DN, Bastos AC, Pedromônico MRM, de Almeida-Filho N, Barreto ML. Ambiente familiar e desenvolvimento cognitivo infantil: uma abordagem epidemiológica. Rev Saúde Pública. 2005;39(4):606-11.

Diaz AG, Schwarcz R. Mortalidad materno-infantil en las Amercias y el enfoque de risco. Montevideo: Publicación científica CLAP. 1987; 1149.

Baltieri L, Santos DCC, Gibim NC, Souza CT, Batistela ACT, Tolocka RE. Desempenho motor de lactentes frequentadores de berçários em creches públicas. Rev Paul Pediatr 2010; 28(3): 283-289.

Barnhart RC, Davenport MJ, Epps SB, Norquist VM. Developmental coordination disorder. Journal of the American Physical Therapy Association, Chicago 2003;83:722-731.

Barnett A. et al. Neurological and perceptual-motor outcome at 5-6 years of age in children with neonatal encephalopathy: relationship with neonatal brain MRI. Neuropediatrics 2002;33(5):242-248. 
Beccaria E, Martino M, Briatore E, Podestà B, Pomero G, Micciolo R, Espa G, Calzolaria S. Poor repertoire General Movements predict some aspects of development outcome at 2 years in very preterm infants. Early Hum Dev. 2012;88(6):393-6.

Biscegli TS, Polis LB, Santos LM, Vicentin M. Avaliação do estado nutricional e do desenvolvimento neuropsicomotor em crianças freqüentadoras de creche. Rev Paul Pediatr. 2007; 25(4): 337-342

Blank R, Smits-Engelsman B, Polatajko H, Wilson P. European Academy for Childhood Disability (EACD): Recommendations on the definition, diagnosis and intervention of developmental coordination disorder (long version). Developmental Medicine \& Child Neurology 2012;54(1):54-93

Bhutta AT, Cleves MA, Casey PH, et al: Cognitive and behavioral outcomes of school-aged children who were born preterm. A meta analysis. J Am Med Assoc 2002;288:728-737.

Braga LW. Family participation in the rehabilitation of the child with brain injury. Rev Med Child Neurol 2010;52(3):1-2.

Bradley RH, Corwyn RF. Socioeconomic status and child development. Annu Rev Psychol. 2002;53:371-99.

Cantell MH, Smyth MM, Ahonen TP. Clumsiness in adolescence: educational, motor, and social outcomes of motor delay detected at 5 years. Adapted Physical Activity Quarterly, Champaign 1994;11:115-129.

Cantell MH, Smyth M, Ahonen TP. Two distinct pathways for developmental coordination disorder: persistence and resolution. Human Movement Science, Amsterdam 2003;22:4-5. 
Clark, CA, Woodward, LJ, Horwood, LJ, Moor, S. Development of emotional and behavioral regulation in children born extremely preterm and very preterm: Biological and social influences. Child Development. 2008;79(5):1444-1462.

Cousins M, Smyth MM. Developmental coordination impairments in adulthood. Human Movement Science 2003;22:433-459.

Dewey D, Wilson BN, Crawford SG, Kaplan BJ. Comorbidity of developmental coordination disorder with ADHD and reading disability. Journal of the International Neuropsychological Society, Cambridge 2000;6:152.

Diament A, Cypel S. Neurologia Infantil. São Paulo: Atheneu, 2005.

Delobel-Ayoub $\mathrm{M}$ et al. Behavioral outcome at 3 years of age in very preterm infants: the EPIPAGE study. 2006; 117(6):1996-2005.

Edgin JO, Inder TE, Anderson PJ, Hood KM, Clark CA, Woodward LJ. Executive functioning in preschool children born very preterm: relationship with early white matter pathology. J Int Neuropsychol Soc. 2008;14(1):90-101.

Edwards $\mathrm{J}$ et al. Developmental Coordination Disorder in School-Aged Children Born Very Preterm and/or at Very Birth Weight: A Systematic Review. Journal of Developmental Behavioral Pediatrics 2011;32:678-687.

Einspieler C, Prechtl HFR, Ferrari F, Cioni G, Bos AF. The qualitative assessment of general movements in preterm, term and young infants - review of the methodology. Early Hum Dev 1997;50:47-60.

Einspieler C, Prechtl HF. Prechtl assessment of general movements: a diagnostic tool for the functional assessment of the young nervous system. Ment Retard Dev Disabil Res Rev. 2005; 11(1):61-67. 
Einspieler C, Prechtl HF, Bos AF, Ferrari F, Cioni G. Prechtl's Method on the Qualitative Assessment of General Movements in Preterm, Term and Young Infants. London, United Kingdom: Mac Keith Press; 2004.

Einspieler C, Marschik PB, Pansy J, Scheuchenegger A, Krieber M, Yang H, et al. The general movement optimality score: a detailed assessment of general movemtns during preterm and term age. Dev Med Child Neurol. 2015;58:286-291.

Einspieler C, Bos AF, Libertus ME, Marschik PB. The General Movement Assessment helps us to identify preterm infants at risk for cognitive dysfunction. Front Psychol. 2016c;7:406.

Einspieler C, Sigafoos J, Bartl-Pokomy KD, Landa R, Marschik PB, Bölte S. Highlighting the first 5 months of life: General movements in infantis later diagnosed with autism spectrum disorder or Rett syndrome. Res Autism Spectr Dis 2014;8:286291.

Ellinoudis, T. Reliability and validity of age band 1 of the movement assessment battery for children - second edition. Research in Developmental Disabilities $2011 ; 32(3): 1046-1051$.

Ermisch J, Francesconi M. The effect of parental employment on child schooling. J Appl Econom. 2013;28:796-822.

Fawke J. Neurological outcomes following preterm birth. Semin Fetal Neonatal Med 2007;12:374-382.

Ferrari F, Cioni G, Einspieler C, Roversi F, Bos AF, Paolicelli PB, Ranzi A, Prechtl HFR. Cramped Syncronized General Movements in Preterm Infants as an Early Marker for Cerebral Palsy. Arch PediatrAdolesc Med 2002;156:460-467. 
Foulder-Hughes LA, Cooke RW. Motor, cognitive and behavioural disorders in children born very preterm. Dev Med Child Neurol 2003;45(2)97-103.

Geuze R, Borger H. Children who are clumsy: five years late. Adapted Physical Activity Quarterly, Champaign 1993;10:10-21.

Goyen TA, Lui K. Developmental coordination disorder in "apparently normal" schoolchildren born extremely preterm. Arch Dis Child 200994(4)298-302.

Green D, Baird G. DCD and overlapping conditions. In: Sugden DA, Chambers ME, eds. Children With Developmental Coordination Disorder. London: Whurr, 2005:9311.

Greene MM, Patra K, Silvestri JM, Nelson MN. Re-evaluating preterm infants with the Bayley III: Patterns and predictors of change. Research in Developmental Disabilities 2013;34:2107-2117.

Green D, Baird G, Barnett AL, Henderson L, Huber J, Henderson SE. The severity and nature of motor impairment in Asperger's syndrome: a comparison with specific developmental disorder of motor function. Journal of child psychology and psychiatry 2002;43(5):655-668.

Groen SE, Blècourt ACE, Postema K, Hadders-Algra M. General movements in early infancy predict neuromotor development at 9 to 12 years of age. Developmental Medicine \& Child Neurology 2005; 45:731-738.

Hack M. Young adult outcomes very-low-birth-weigth children. Seminars in Fetal \& Neonatal Medicine 2006;11:127-137.

Prado, MS. Tradução e adaptação cultural do Developmental coordination disorder Questionnaire (DCDQ). 81f. Dissertação (Mestrado Ciências da Reabilitação) - 
Escola de Educação Física, Fisioterapia e Terapia Ocupacional, Universidade Federal de Minas Gerais, Belo Horizonte. 2007:81f.

Prechtl HFR. General movement assessment as a method of developmental neurology: new paradigms and their consequences. Developmental Medicine \& Child Neurology 2001;43:836-842.

Henderson SE, Sugden DA, Barnett AL. Movement assessment battery for children2 (2nd ed.) London: Harcourt Assessment, 2007.

Jongmans, MJ, Mercuri, E, Dubowitz, LMS, Henderson SE. Perceptual-motor difficulties and their concomitants in six-year-old children born prematurely. Human Movement Science 1998;17(25):629-653.

Kaplan B, Crawford S, Cantell M, Kooistra L, Dewey D. Comorbidity, co-occurrence, continuum: What's in a name? Child Care Health Dev 2006;32(6):723-31.

Kirby A, Sugden D, Purcell C. Diagnosing developmental coordination disorders. Archives of disease in childhood 2014;99(3):292-296.

Bruggink JLM, Einspieler C, Butcher PR, Stremmelaar EF, Prechtl HFR, BOS AF. Quantitative aspects of the early motor repertoire in preterm infants: Do they predict minor neurological dysfunction at school age? Early Human Development. 2009;85(25).

Linhares MBM, Carvalho AEV, Machado C, Martinez FE. Desenvolvimento de bebês nascidos pré-termo no primeiro ano de vida. Paidéia 2003; 13(25): 59-72. 
Losse A, Henderson SE, Elliman D, Hall D, Knight E, Jongmans M. Clumsiness in children-do they grow out of it? A 10-year follow-up study. Developmental Medicine \& Child Neurology, London 1991;33:55-68.

Magalhães LC, Rezende FCA, Magalães CM, Aalbuquerque PDR. Análise comparativa da coordenação motora de crianças nascidas a termo e pré-termo, aos 7 anos de idade.; Rev. Bras. Matern. Infant. Recife 2009;9(3):293-300.

Maggi EF. Desenvolvimento cognitivo e funcional de crianças nascidas pré-termo e a termo, de níveis sociais diferentes, aos quatro anos de idade. Dissetação de Mestrado. Universidade Federal de Minas Gerais, Faculdade de Medicina 2012.

Marlow N, Hennessy EM, Bracewell MA, Wolke D. Motor and executive function at 6 years of age after extremely preterm birth. Pediatrics 2007;120:793-804.

Miranda TB, Beltrame TS, Cardoso FL. Desempenho motor e estado nutricional de escolares com e sem transtorno do desenvolvimento da coordenação. Rev Bras Cineantropom Desempenho Hum. 2011,13(1):59-66

Missiuna C, Mol S, King S, King G e Law M. A trajectory of Troubles: Parent's Impressions of the Impact of Developmental Coordination Disorder. Physical \& Occupational Therapy in Pediatrics 2007;27:81-101.

Missiuna C, Cairney J, Pollock N, Russel D, Macdonald K, Cousins M, Veldhuizen S, Schmidt L. A staged approach for identifying children with developmental coordination disorder from the population. Research in Developmental Disabilities, 2011;32(2):549-559.

Missiuna, C, Mclean, J, DeLaat D, Egan M, Soucie H. Description of children identified by physicians as Having developmental coordination Disorder. Developmental Medicine \& Child Neurology, 2008;50:839-844. 
Moraes MW, Weber APR, Santos MCO, Almeida FA. Teste de Denver II: avaliação do desenvolvimento de crianças atendidas no ambulatório do Projeto Einstein na Comunidade de Paraisópolis. Einstein 2010;8(2):149-153.

Nascimento RO, Ferreira LF, Goulardins JB, Freudenhem AM, Marques JCB, Casella EB, Oliveira JA.Health-related physical fitness children with severe and moderate developmental coordination disorder.Research in Developmental Disabilities 2013;34(11):4222-4231.

Noble Y, Boyd RN. Neonatal assessments for the preterm infant up to 4 months corrected age: a systematic review. Dev Med Child Neurol 2012;54:129-139.

Orton J, Spittle A, Doyle L, Anderson P, Boyd R. Do early intervention programmes improve cognitive and motor outcomes from preterm infantis after discharges? A systematic review. Developmental Medicine \& Child Neurology 2009;51:851-859.

Palmer FB. Strategies for the early diagnosis of cerebral palsy. J Pediatr 2004;145:811.

Pampel FC, Krueger PM, Denney JT. Socioeconomic Disparities in Health Behaviors. Annual Review of Sociology. 2010; 36:349-370.

Parizzi MBF, Fonseca JGM. O bebê e o tempo. Revista Linguagem, São Paulo 2015;1: 23.

Payne V, Isaacs L. Desenvolvimento motor humano: uma abordagem vitalícia. Rio de Janeiro: Guanabara: Koogan; 2007.

Pedraza DF, Menezes TN. Fatores de risco do déficit de estatura em crianças préescolares: estudo caso-controle. Ciênc Saúde Coletiva. 2014; 19(5): 1495-1502. 
Piccolo LR, Falceto OG, Fernandes CL, Levandowski DC, Grassi-Oliveira R, Salles JF. Variáveis psicossociais e desempenho em leitura de crianças de baixo nível socioeconômico. Psicol Teor Pesq. 2012;28:389-98.

Prechtl HFR, Einspieler C, Cioni G, Bos AF, Ferrari, Sontheimer D. Na early marker for neurlogicaldéficits after perinatal brain lesions. Lancet 1997;349:1361-1363.

Polatajko HJ, Fox M, Missiuna C. An international consensus on children with developmental coordination disorder. Canadian Journal Occupational Therapy. 1995;62:3-6.

Rademaker KJ, Lam JN, Van Haastert IC, Uiterwaal CS, Lieftink AF, Groenendaal F, et-al. Larger corpus callosum size with better motor performance in prematurely born children. Semin Perinatol. 2004;28:279-87.

Resegue R, Puccini RF, Silva EMKS. Fatores de risco associado a alterações no desenvolvimento da criança. Pediatria. 2007; 29(2):117-128.

Ribeiro DG, Perosa GB, Padovani FHP. Fatores de risco para o desenvolvimento de crianças atendidas em Unidades de Saúde da Família, ao final do primeiro ano de vida: aspectos sociodemográficos e de saúde mental materna. Ciência \& Saúde Coletiva 2014; 19(1):215-226.

Romeo DM, Brogna C, Sini F, Romeo MG, Cota F, Ricci D. Early psychomotor development of low-risk preterm infants: influence of gestational age and gender. Eur J Paediatr Neurol. 2016;20(4):518-23

Rosa Neto F, Santos APM, Xavier RFC, Amaro KNA. Importância da avaliação motora em escolares: análise da confiabilidade da Escala de Desenvolvimento Motor. Rev Bras Cineantropom Desempenho Hum. 2010; 12(6):422-427. 
Rugolo LMSS. Crescimento e desenvolvimento a longo prazo do prematuro extremo. J Pediatr 2005;81:101-10.

Sameroff AJ. Environmental context of child development. J Pediatr. 1986;109(1):192-9.

Samsom JF, De Groot L. Study of a group of extremely preterm infants (25-27 weeks): how do they function at 1 year of age? Journal of Child Neurology. 2001;16(11)832-837.

Santos S, Dantas L, Oliveira J. Desenvolvimento motor de crianças, de idosos e de pessoas com transtornos da coordenação. Rev Paul Educ Fís. 2004;18:33-44.

Santos RS, Araújo AP, Porto MA. Early Diagnosis of Abnormal Development of Preterm Newborns: Assessment Instruments. J Pediatr. 2008;84:289-99.

Santos VAPD, Contreira A R, Caruzzo NM, Passos PCB, Vieira JLL. Desordem coordenativa desenvolvimental: uma análise do estado nutricional e nível socioeconómico. Motricidade 2015;11(1):78-86.

Schoemaker MM, Kalverboer AF. Social and affective Problems of Children Who Are Clumsy: How Early Do They Begin? Adapted Psysical Activity Quarterly, Champaing 1994;11:130-140.

Schoemaker MM, Flapper B, Verheij NP, Wilson BN, Reinders-Messelink HA, Kloet A. Evaluation of the Developmental Coordination Disorder Questionnaire as a screening instrument. Developmental Medicine \& Child Neurology 2006;48(8):668673.

Sistema de Informação sobre Nascidos Vivos - SINASC, 2017. MS/SVS/DASIS. 
Silva J, Beltrame TS. Indicativo de transtorno do desenvolvimento da coordenação de escolares com idade entre 7 e 10 anos. Rev Bras Ciênc Esporte. 2013; 35(1):314.

Smits-Engelsman BC, Niemeijer AS, Van Waelverde $H$. Is the Movement Assessment Battery od Children-2nd edition a reliable instrument to measure motor performance in 3 year old children? Res Dev Disabil 2011;32(4):1370-7.

Spittle AJ, Spencer-Smith MM, Cheong JL, Eeles AL, Lee KJ, Anderson PJ e Doyle LW. General Movements in very preterm and neurodevelopment at 2 and 4 years. Pediatrics 2013;132(2)452-8.

Spittle AJ, Walsh JM, Potter C, Mcinnes E, Olsen J, Lee KJ, Anderson PJ, Doyle LW, Cheong JLY. Neurobehaviour at term- equivalent age and neurodevelopmental outcomes at 2 years in infants born moderate-to-late preterm. Developmental Medicine \& Child Neurology 2017;59:207-215.

Smits-Engelsman B, Schoemaker M, Delabastita T, Hoskens J, Geuze R. Diagnostic criteria for DCD: Past and future.Human Movement Science 2015;42:293-306.

Sugden, D, Chambers ME. Stability and change in children with Developmental Coordination Disorder. Child: Care, Health and Development. 2006;33(5):520-528.

Sullivan MC, McGrath MM. Perinatal morbidity, mild motor delay, and later school outcomes. Dev Med Child Neurol 2003;45:104-12.

Tani G. Educação física na pré-escola e nas quatro primeiras séries do ensino de primeiro grau: uma abordagem de desenvolvimento. Kinesis. 1987;3:19-41. 
Tseng MH, Fu CP, Wilson BN, Hu FC. Psychometric properties of a Chinese version of the Developmental Coordination Disorder Questionnaire in community-based children. Research in Developmental Disabilities. 2010;31:33-45.

Vaivre-Douret L. Developmental coordination disorders: state of art. NeurophysiolClin 2014;44(1):13-23.

Valentini NC, Clark JE, Whitall J. Developmental co-ordination disorder in socially disadvantaged Brazilian children.Child: care, health and development 2015;41(6):970-979.

Valentini NC, Ramalho MH, Oliveira MA. Movement Assessment Battery for Children-2: Translation, reliability, and validity for Brazilian children. Research in Developmental Disabilities 2013;35(3):733-740.

Wann J. (commentary) Current approaches to intervention in children with developmental coordination disorder. Developmental Medicine \& Child Neurology 2007;19:405-405.

Willrich A, Azevedo CCF, Fernandes JO. Desenvolvimento motor na infância: influência dos fatores de risco e programas de intervenção. Rev Neurocienc. 2009; 17(1): 51-56.

Woodward LJ, Edgin JO, Thompson D, Inder TE. Object working memory deficits predicted by early brain injury and development in the preterm infant. Brain 2005;128:2578-87.

Zahed-Cheikh M, Brévaut-Malaty V, Busuttil M, Monnier AS, Roussel M, Gire C. Comparative analysis of perinatal and postnatal factors, and general movement in extremely preterm infantis. Brain Dev 2011;33(8):656-65. 
Zwicker JG, Harris SR, Klassen AF. Quality of life domains affected in children with developmental coordination disorder: a systematic review. Child: care, health and development 2013;39(4):562-580.

Zwicker, JG, Missiuna, C, Harris, SR, Boyd, LA. Developmental Coordination Disorder: A Pilot Diffusion Tensor Imaging Study. Pediatric Neurology, 2012; 80(46):162-167. 


\section{ANEXOS}

ANEXO 1 - Termo de Consentimento

TERMO DE CONSENTIMENTO LIVRE E ESCLARECIDO

DADOS DE IDENTIFICAÇÃO DO SUJEITO DA PESQUISA OU RESPONSÁVEL LEGAL

1.NOME::

DOCUMENTO DE IDENTIDADE № :

SEXO:.$M() F()$

DATA NASCIMENTO:

ENDEREÇO

№

APTO:

BAIRRO: CIDADE

CEP: .TELEFONE: DDD ..)

2.RESPONSÁVEL LEGAL

NATUREZA (grau de parentesco, tutor, curador etc.)

DOCUMENTO DE IDENTIDADE :

SEXO: $M() \quad F()$

DATA NASCIMENTO.: .....................

ENDEREÇO:

№ APTO:

BAIRRO: CIDADE:

CEP: TELEFONE: DDD

DADOS SOBRE A PESQUISA

1. TÍTULO DO PROTOCOLO DE PESQUISA: "Predição de risco para dificuldades motoras em prematuros com idade pré-escolar pelo General Movement Assessment“

PESQUISADOR : Renata Hydee Hasue

CARGO/FUNÇÃO: Professor Doutor INSCRIÇÃO CONSELHO REGIONAL № 21370F

2. AVALIAÇÃO DO RISCO DA PESQUISA:

$\begin{aligned} \text { RISCO MÍNIMO } \quad X & \text { RISCO MÉDIO } \square \\ \text { RISCO BAIXO } & \text { RISCO MAIOR } \square\end{aligned}$


Rubrica do sujeito responsável:

Rubrica do pesquisador:

3. DURAÇÃO DA PESQUISA: 24 meses.

\section{HOSPITAL UNIVERSITÁRIO DA USP - UNIVERSIDADE DE SÃO PAULO}

Essas informações estão sendo fornecidas para sua participação voluntária neste estudo, que tem como objetivo avaliar o desenvolvimento infantil de crianças nascidas no Hospital Universitário da USP.

Serão feitos dois exames: para saber se o desenvolvimento motor da criança está normal. Estes testes são baseados em brincadeiras como argola, boneca, carrinho, quebra-cabeça, bola, saltos e outros brinquedos já usados pelas crianças de acordo com cada idade para saber se seus movimentos, equilíbrio, a inteligência e a fala estão normais. Todos os testes serão realizados com a criança em posição confortável, em uma sala arejada e bem iluminada e ela não será obrigada a realizar os procedimentos caso não queira. Estes testes todos são necessários para saber se estas crianças já têm, ou podem ter, problemas relacionados ao desenvolvimento no futuro, e poderemos indicar o tratamento mais adequado para cada criança.

Durante a pesquisa a criança poderá continuar com todas as suas atividades, e nenhuma atividade que a criança venha a desenvolver poderá interferir nos resultados. Os profissionais responsáveis pela pesquisa estarão a disposição para qualquer dúvida e esclarecimento. O principal investigador é a Profa. Dra. Renata Hydee Hasue, que pode ser encontrada no endereço Rua Cipotânea, no 51, Cidade Universitária. Telefone(s) 3091-8423. Caso tenha qualquer dúvida sobre a ética da pesquisa, entre em contato com Comitê de Ética em Pesquisa do Hospital Universitário da USP (CEPHU/USP): Av. Prof. Lineu Pestes, 2565 - Cidade Universitária - CEP: 05508-000 - São Paulo - SP Telefone: (11) 3091-9457 - Fax: (11) 3091-9479 - E-mail: cep@hu.usp.br.

A qualquer momento, a criança ou o responsável está livre para deixar de participar do estudo, sem qualquer prejuízo à continuidade de seu tratamento na Instituição.

As informações obtidas serão analisadas em conjunto com outros pacientes, não sendo divulgada a identificação de nenhum paciente. A criança ou o responsável terá direito de ser atualizado sobre os resultados parciais das pesquisas, quando em estudos abertos, ou de resultados que sejam do conhecimento dos pesquisadores. Não há despesas pessoais para o participante em qualquer fase do estudo, incluindo exames e consultas. Também não há compensação financeira relacionada à sua participação. Se existir qualquer despesa adicional, ela será absorvida pelo orçamento da pesquisa. Os dados e o material coletado somente serão utilizados para esta pesquisa.

Rubrica do sujeito responsável:

Rubrica do pesquisador: 
Acredito ter sido suficientemente informado a respeito das informações que li ou que foram lidas para mim, descrevendo o estudo "Predição de risco para dificuldades motoras em prematuros com idade pré-escolar pelo General Movement Assessment“"

Eu discuti com a Profa. Dra. Renata Hydee Hasue sobre a minha decisão em participar nesse estudo. Ficaram claros para mim quais são os propósitos do estudo, os procedimentos a serem realizados, seus desconfortos e riscos, as garantias de confidencialidade e de esclarecimentos permanentes. Ficou claro também que minha participação é isenta de despesas e que tenho garantia do acesso a tratamento hospitalar quando necessário. Concordo voluntariamente em participar deste estudo e poderei retirar o meu consentimento a qualquer momento, antes ou durante o mesmo, sem penalidades ou prejuízo ou perda de qualquer benefício que eu possa ter adquirido, ou no meu atendimento neste Serviço.

Assinatura do paciente/representante legal

Data

11

Assinatura da testemunha

Data

11

para casos de pacientes menores de 18 anos, analfabetos, semi-analfabetos ou portadores de deficiência auditiva ou visual.

\section{(Somente para o responsável do projeto)}

Declaro que obtive de forma apropriada e voluntária o Consentimento Livre e Esclarecido deste paciente ou representante legal para a participação neste estudo.

Assinatura do responsável pelo estudo

Data

Data

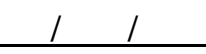




\section{ANEXO 2 - Aprovação Comitê de Ética}

S\$o Paulo, 23 de abril de 2018.

$I^{\text {eniaj }} \cdot S^{\text {riaj }}$.

Dra. Rewata Hydee Hasue

Pesyuisador(a) responsável

Departamento de Fisioterapia, Fonosudiologia e Terapia Ocupacional

Faculdade de Medicina

UNIVERSIDADE DE SÃO PAULO

REFERENTE- Projeto de Pesquisa "Caracterizanaso dos riscos para o Transtomo do Desenvolvimento e da Coordenaçồ (TDC) em crianças coon idade pré-escolar ( 3 a 5 anos) Pesquisadora executante: Carolina Barbosa de Souza

CANE: 83899617.2 .3001 .0076

Registro CEP-HU/USP: $1704 / 18$

Precado(a) Senhor(a)

O Comitê de Ėtica em Pesquisa do Hospital Universitário da Universidade de São Paulo, em reuniầo ondinária realizada m dia 20 de abril de 2018 anatisou o Projeto de Pesquisa acima citado, consideranbo-D camo APROVADO, bem como o seu Termo de Consentimento Livre e Esclarecido.

Lembramos que cabe ao pesquisador elaborar $\mathrm{e}$ apresentar a este Comitê, relatớios parciais semestrais e final, de acondo com a Resoluģo $n^{2} 466 / 2012$ do Conselho Nacional de Swide, inciso XI 2, letra " $\mathrm{d}$ ".

O primeiro relatório está previsto para 20 de outubro de 2018.

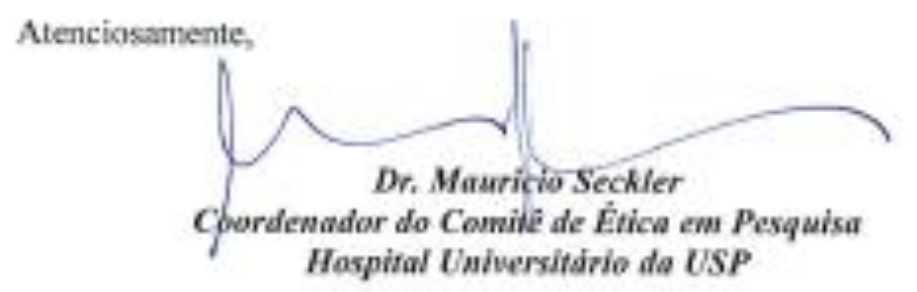




\section{ANEXO 3 - Termo de Assentimento}

Nome:

Idade:

\section{VAMOS BRINCAR?}

Você irá fazer:

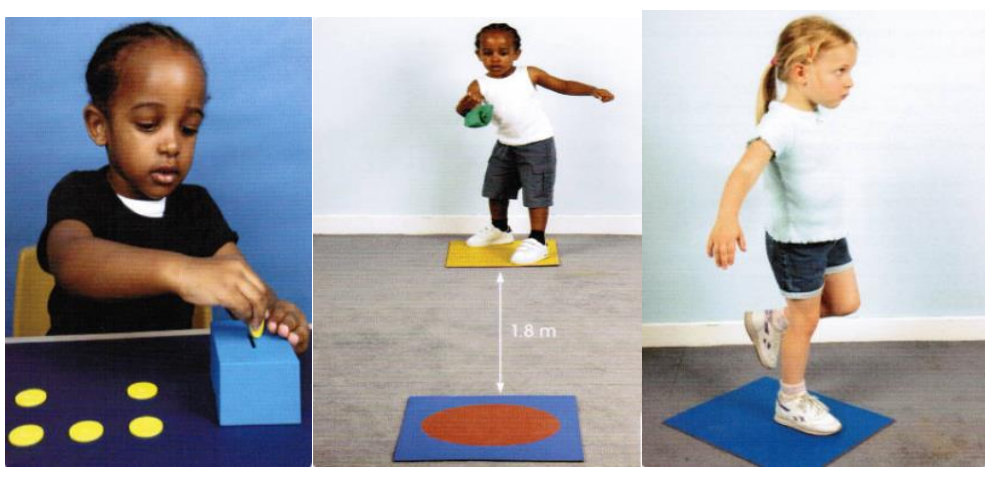

$\underline{\text { E eu vou olhar você fazer e contar o tempo! }}$

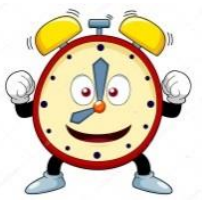

Se você ficar cansado, pode parar quando quiser!

Seus pais já sabem que eu vou te convidar para isso e me deixaram conversar com você.

\section{Você quer participar?}

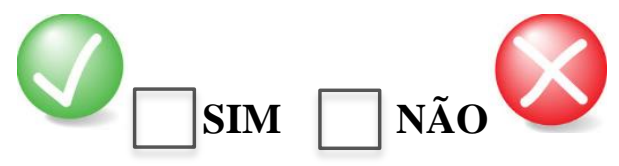

São Paulo,

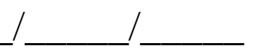

Rubrica da criança ou representante legal:

Rubrica do pesquisador:

Observação: A criança participante da pesquisa não é totalmente capaz de ler o Termo de Assentimento, portanto, as informações descritas no documento são explicadas oralmente. A criança tem total liberdade para aceitar ou não participar do estudo, demonstrando sua decisão ao assinalar "sim", apenas pintando o quadrado correspondente e se conseguir rubricar seu nome. 


\section{FICHA DE ANAMNESE - DADOS DO PARTICIPANTE, MATERNOS E} SOCIODEMOGRÁFICOS

\section{IDENTIFICAÇÃO DA CRIANÇA:}

Nome:

Data de nascimento:

Sexo: ( ) F ( ) M Matrícula HU:

\section{DADOS MATERNOS A GESTACIONAIS:}

Nome da mãe:

Data de nascimento:

Houve intercorrências durante a gestação? (HAS, DM, infecções, corrimento vaginal, sangramento vaginal, alergias)

Fez uso de alguma medicação durante a gestação?

Fez uso de álcool, cigarro ou drogas?

Sorologias:

Houve tentativa de aborto durante a gestação?

( ) Sim ( )Não

Foi realizado pré-natal?

Número de consultas: Local:

Tipo de parto?

( ) Normal

( ) Cesárea

( ) Fórceps

Tempo de trabalho de parto:

Intercorrências durante o parto:

SOBRE A CRIANÇA:

Idade gestacional: Apgar: $1^{\circ} \mathrm{min}$ : $5^{\circ} \mathrm{min}:$ $10^{\circ} \mathrm{min}:$ 
Peso ao nascimento:

Necessidade de suporte ventilatório?

( ) Sim ( )Não

Qual? Quanto tempo?

Número de internações?

Período da última internação:

Motivo:

Realiza acompanhamento médico? Quais?

Diagnósticos médicos existentes:

Já realizou fisioterapia? ( ) Sim ( ) Não Local?

Por quanto tempo?

Já realizou algum outro tipo de acompanhamento?

( ) $\operatorname{Sim}($ ) Não

Qual?

A criança freqüenta a escola? ( ) Sim ( ) Não Qual?

\section{CONDIÇÕES SOCIO-ECONÕMICAS DA FAMÍLIA}

Pais são casados ou moram juntos?

Possui irmãos/irmãs?

Condições da moradia:

( ) Casa ( ) Apartamento / ( ) Própria ( ) Alugada

Escolaridade: mãe: pai:

A mãe trabalha? ( ) Sim ( ) Não Qual função?

O pai trabalha? ( ) Sim ( ) Não Qual função?

Qual a renda familiar?

Região de moradia? UBS de referência: 
ANEXO 5 - MABC-2

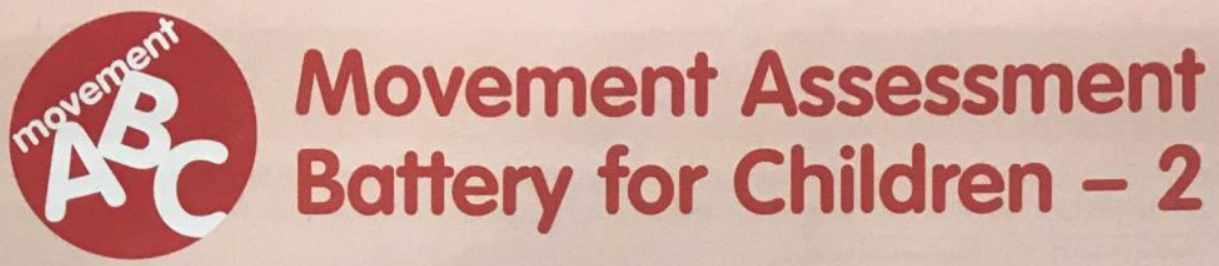

Test Record Form Age Band 1 (3-6 years)

\begin{tabular}{|c|c|c|c|c|}
\hline Name: & & \multicolumn{2}{|c|}{ Gender: M/F } & \\
\hline \multicolumn{5}{|l|}{ Home address: } \\
\hline \multicolumn{2}{|l|}{ School: } & \multicolumn{2}{|c|}{ Class/year/grade: } & \\
\hline \multicolumn{5}{|l|}{ Assessed by: } \\
\hline \multicolumn{5}{|l|}{ Referral source: } \\
\hline \multicolumn{2}{|l|}{ Preferred (writing) hand: } & Year & Month & Day \\
\hline & Date tested & & & \\
\hline \multirow{2}{*}{$\begin{array}{l}\text { Movement ABC-2 } \\
\text { Checklist completed? Y/N }\end{array}$} & Date of birth & & & \\
\hline & Chronological age & & & \\
\hline
\end{tabular}

Item Scores and Equivalent Standard Scores

\begin{tabular}{|l|l|l|l|}
\hline $\begin{array}{l}\text { Item } \\
\text { code }\end{array}$ & Name of item & $\begin{array}{l}\text { Raw score } \\
\text { (best attempt) }\end{array}$ & $\begin{array}{l}\text { Item Standard } \\
\text { Score }\end{array}$ \\
\hline \multirow{2}{*}{ MD 1* } & $\begin{array}{l}\text { Posting Coins } \\
\text { preferred hand }\end{array}$ & & \\
\hline & $\begin{array}{l}\text { Posting Coins } \\
\text { non-pref hand }\end{array}$ & & \\
\hline MD 2 & Threading Beads & & \\
\hline MD 3 & Drawing Trail 1 & \\
\hline A\&C 1 & $\begin{array}{l}\text { Catching } \\
\text { Beanbag }\end{array}$ & \\
\hline A\&C 2 & $\begin{array}{l}\text { Throwing Beanbag } \\
\text { onto mat }\end{array}$ & \\
\hline \multirow{2}{*}{ Bal 1* } & $\begin{array}{l}\text { One-Leg Balance } \\
\text { best leg }\end{array}$ & \\
\hline & $\begin{array}{l}\text { One-Leg Balance } \\
\text { other leg }\end{array}$ & \\
\hline Bal 2 & $\begin{array}{l}\text { Walking Heels } \\
\text { Raised }\end{array}$ & \\
\hline Bal 3 & $\begin{array}{l}\text { Jumping } \\
\text { on Mats }\end{array}$ & \\
\hline
\end{tabular}

Three Component Scores

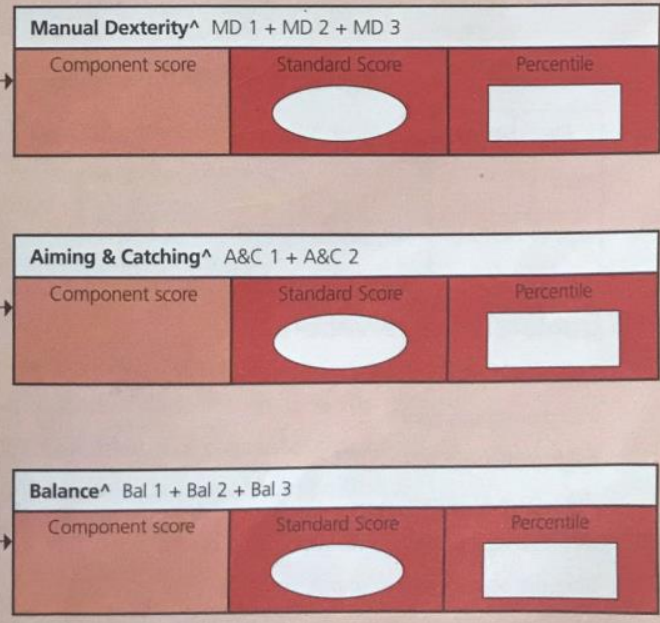

"In each case sum the item standard scores.

Total Test Score

Sum of 8 item standard scores:

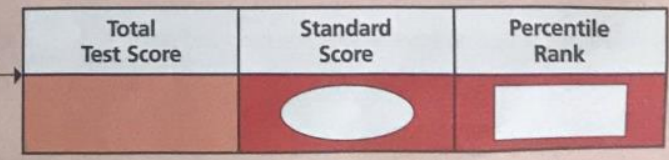

For Posting Coins and One-Leg Balance, look up standard score each limb, add these and divide by 2 . If the result is above 10 , round up; if below 10 , round down. 